Pacific Journal of Mathematics

HOMOTOPY CONDITIONS WHICH DETECT SIMPLE 


\title{
HOMOTOPY CONDITIONS WHICH DETECT SIMPLE HOMOTOPY EQUIVALENCES
}

\author{
T. A. Chapman
}

\begin{abstract}
Let $X, Y$, and $K$ be compact polyhedra, let $p: Y \times K \rightarrow Y$ be the projection map, and let $f: X \rightarrow Y \times K$ be a homotopy equivalence which has a homotopy inverse $g: Y \times K \rightarrow X$ along with homotopies $f g \simeq \mathrm{id}, g f \simeq \mathrm{id}$ such that $p(f g \simeq \mathrm{id})$ and $p f(g f \simeq \mathrm{id})$ are small homotopies. In this paper we prove that if $\pi_{1}$ of each component of $K$ is free abelian, then $f$ must be a simple homotopy equivalence.
\end{abstract}

1. Introduction. All spaces in this paper will be locally compact, separable and metric, and a proper map is a map for which preimages of compacta are compact. The following is the main technical definition of this paper. If $\alpha$ is an open cover of $Y$, then a proper map $f: X \rightarrow Y$ is said to be an $\alpha$-equivalence provided that there is a map $g: Y \rightarrow X$, an $\alpha$-homotopy of $f \circ g: Y \rightarrow Y$ to the identity, and an $f^{-1}(\alpha)$-homotopy of $g \circ f: X \rightarrow X$ to the identity. Here $f^{-1}(\alpha)=\left\{f^{-1}(U) \mid U \in \alpha\right\}$, and a $\beta$-homotopy is a homotopy for which the track of each point lies in some element of $\beta$ (see $\S 2$ ).

In [14] Ferry used $Q$-manifolds to prove the following result:

If $Y$ is a polyhedron, then there is an open cover $\alpha$ of $Y$ so that for any polyhedron $X$ and $\alpha$-equivalence $f: X \rightarrow Y, f$ must be a simple homotopy equivalence.

(For the definition of a simple homotopy equivalence (s.h.e.) for compact polyhedra we refer the reader to [24], and for noncompact polyhedra we refer to [19], where the designation infinite s.h.e. is used.) The above result represents the most general homotopy conditions that the author knows of which detect s.h.e.'s. It easily implies half of the Classification Theorem from $Q$-manifold theory [7, p. 88], which gives a homeomorphism condition which detects s.h.e.'s (see Theorem 2 below). On the other hand it follows from [16] that any cell-like map of polyhedra must be an $\alpha$-equivalence, for every $\alpha$. Therefore the above result implies that every cell-like map of polyhedra is a s.h.e., thus recapturing the main result of [5].

The purpose of this paper is to generalize the above result, while at the same time giving a proof which does not rely upon $Q$-manifold theory. In what follows $K$ will be a compact polyhedron for which each Whitehead group $\mathrm{Wh}\left(K \times T^{n}\right)$ vanishes, where $T^{n}$ is the $n$-torus $\left(T^{0}=\right.$ \{point $\left.\}\right)$. This includes, for example, all polyhedra $K$ for which $\pi_{1}$ of each component of $K$ is free abelian or 
(more generally) poly $Z$ [13]. Here is our main result.

THEOREM 1. For any polyhedron $Y$ with projection map p: $Y \times K \rightarrow Y$, there exists an open cover $\alpha$ of $Y$ so that if $X$ is a polyhedron and $f: X \rightarrow Y \times K$ is a $p^{-1}(\alpha)$-equivalence, then $f$ is a s.h.e. Moreover, $\alpha$ depends only on $Y$.

It is clear that we cannot completely remove the $\pi_{1}$ restriction on $K$, for if $Y$ is a point we can choose compact polyhedra $X$ and homotopy equivalences $f: X \rightarrow K$ which are not s.h.e.'s [11, p. 98]. Note that Theorem 1 implies that any homeomorphism between polyhedra is a s.h.e., thus giving another proof of the topological invariance of simple homotopy type for polyhedra [4].

The proof of Theorem 1 that we give here uses no $Q$-manifold theory. We will work entirely in the PL category of polyhedra, and we rely on torus geometry in the spirit of [21]. The niceness condition on $\pi_{1}$ of each component of $K$ is used to conclude that some obstructions encountered in certain projective class groups and Whitehead groups vanish. It would be interesting to know if the $\pi_{1}$ condition on $K$ could be replaced by the assignment of a torsion to $f: X \rightarrow Y \times K$ in a nice subgroup of the Whitehead group $\mathrm{Wh}(Y \times K)$.

The author feels that Theorem 1 is not the last word in results of this type. It seems probable that the $p^{-1}(\alpha)$-equivalence condition in Theorem 1 can be replaced by a far more general condition on homotopy equivalences $f: X \rightarrow Y$, which would require that there exists a homotopy inverse $g: Y \rightarrow X$ of $f$ such that the homotopies $f \circ g \simeq$ id and $g \circ f \simeq$ id would only "wind around nice elements of $\pi_{1}$."

As an application of Theorem 1 we give a short proof of the following result, which is half of the Classification Theorem of [7, p. 88]. We use $Q$ to represent the Hilbert cube, the countable infinite product of closed intervals. We need nothing at all from $Q$ manifold theory. This is a far cry from the proof of this half of the Classification Theorem given in [7], which uses a lot of $Q$-manifold theory.

THEOREM 2. If $X, Y$ are polyhedra, then a proper map $f$ : $X \rightarrow Y$ is a s.h.e. provided that $f \times$ id: $X \times Q \rightarrow Y \times Q$ is proper homotopic to a homeomorphism.

The other half of the Classification Theorem asserts that given any s.h.e. $f: X \rightarrow Y, f \times$ id: $X \times Q \rightarrow Y \times Q$ is proper homotopic to a homeomorphism. There is a proof of this which uses elementary PL techniques and nothing at all from $Q$-manifold theory [3]. 
We point out that the splitting theorem of $\S 7$ (Theorem 7.2) is in reality the main result of this paper. Once we have established it, Theorem 1 follows by a more-or-less standard argument. Theorem 7.2 is also the main tool used in [8] to investigate the problem of approximating maps of $Q$-manifolds to $Q$-manifold bundles by homeomorphisms.

Finally the author would like to thank Marshall Cohen for an unusually helpful referee's report. Our goal was to produce a paper that would be readable by expert and nonexpert alike, but without the input of the referee we would have certainly failed in both departments.

Here is a list of the sections to follow:

$\S 2$. General preliminaries.

$\S 3$. Preliminaries on equivalences.

$\S 4$. A finiteness result. Here we show that a certain homotopy domination can be extended to a homotopy equivalence in a well-controlled manner. This result is only needed in $\S 5$.

$\S 5$. The handle lemma. Here we use torus geometry to establish the main technical result of this paper. The procedure is similar to that of [6], but the absence of cell-like maps makes the constructions much more complicated. The appearance of the factor $K$ appears to be more of a nuisance than a hindrance.

$\S 6$. The handle theorem. Here the inversion idea of [21] is used to reverse the roles of 0 and $\infty$ in the handle lemma.

$\S 7$. A splitting theorem. Here the handle theorem is applied to prove a general splitting result. This is the form of the handle theorem that is used in $\S 8$.

$\S 8$. Proof of Theorem 1.

$\S 9$. Proof of Theorem 2.

2. General preliminaries. The purpose of this section is to introduce some more notation and to establish some elementary results which will be needed in the sequel.

If $f_{t}: X \rightarrow Y$ is a homotopy, $t \in I=[0,1]$, we use the notation $f_{t}: g \simeq h$ to indicate that $f_{0}=g$ and $f_{1}=h$. If $\alpha$ is an open cover of $Y$, then $f_{t}: X \rightarrow Y$ is an $\alpha$-homotopy provided that the track of each $x \in X,\left\{f_{t}(x) \mid 0 \leqq t \leqq 1\right\}$, lies in some element of $\alpha$. We say that the maps $u, v: X \rightarrow Y$ are $\alpha$-close if each set $\{u(x), v(x)\}$ lies in some element of $\alpha$. We will need the following estimated version of the homotopy extension theorem.

Proposition 2.1. Let $f: X \rightarrow Y$ be a map, $X_{0} \subset X$ be closed, and let $g_{t}: X_{0} \rightarrow Y$ be an $\alpha$-homotopy such that $g_{0}=f \mid X_{0}$. Assume 
either (i) $X_{0}$ and $X$ are ANRs, or (ii) $Y$ is an ANR. Then $g_{t}$ extends an a-homotopy $f_{t}: X \rightarrow Y$ such that $f_{0}=f$.

Proof. We proceed in the usual manner.

(i) Let $r: X \times I \rightarrow(X \times\{0\}) \cup\left(X_{0} \times I\right)$ be a retraction obtained as a composition $r=r_{2} \circ r_{1}$ as follows. For $N$ a small neighborhood of $X_{0}, r_{1}$ is a map of $X \times I$ into $(X \times\{0\}) \cup(N \times I)$ defined by $r_{1}(x, t)=(x, t \varphi(x))$, where $\varphi: X \rightarrow I$ is a map which is 0 on $X-N$ and 1 on $X_{0} . \quad r_{2}$ is a retraction of $(X \times\{0\}) \cup(N \times I)$ onto $(X \times$ $\{0\}) \cup\left(X_{0} \times I\right)$, which exists because $X$ and $X_{0}$ are ANRs. For $N$ close to $X_{0}, r_{2}$ does not move points very far. Define $h:(X \times\{0\}) \cup$ $\left(X_{0} \times I\right) \rightarrow Y$ by $h(x, 0)=f(x)$ and $h(x, t)=g_{t}(x)$, and define $f_{t}: X \rightarrow$ $Y$ by $f_{t}(x)=h \circ r(x, t)$. Note that each track, $\left\{f_{t}(x) \mid 0 \leqq t \leqq 1\right\}$, is a single point for $x \notin N$. For $x \in N$ we may choose $r_{2}$ and $N$ so that the track $\left\{f_{t}(x) \mid 0 \leqq t \leqq 1\right\}$ is close to some track $\left\{g_{t}\left(x^{\prime}\right) \mid 0 \leqq t \leqq 1\right\}$, where $x^{\prime} \in X_{0}$. Thus $f_{t}$ is an $\alpha$-homotopy.

(ii) If $Y$ is an ANR, then there is a small neighborhood $N^{\prime}$ of $(X \times\{0\}) \cup\left(X_{0} \times I\right)$ in $X \times I$ and an extension of $h$ to $h^{\prime}: N^{\prime} \rightarrow Y$, where $h=f_{0} \cup g$ is as above. If $r_{1}$ is as above, we may choose $r_{1}(X \times I) \subset N^{\prime}$, and $f_{t}(x)=h^{\prime} \circ r_{1}(x, t)$ is therefore our desired $\alpha$ homotopy.

If $\alpha, \beta$ are collections of subsets of a set $Y$ and $A \subset Y$, we define

$$
\begin{aligned}
& \operatorname{St}(A, \beta)=\cup\{A \cup U \mid U \in \beta, A \cap U \neq \varnothing\}, \\
& \operatorname{St}^{0}(\alpha, \beta)=\alpha, \\
& \operatorname{St}^{n+1}(\alpha, \beta)=\left\{\operatorname{St}(A, \beta) \mid A \in \operatorname{St}^{n}(\alpha, \beta)\right\} .
\end{aligned}
$$

If $\alpha=\beta$, then we simply write $\operatorname{St}^{n}(\alpha, \beta)=\operatorname{St}^{n}(\alpha)$.

If $f_{t}: X \rightarrow Y$ is a homotopy, $Y$ has a given metric, and $\varepsilon>0$, then we say that $f_{t}$ is an $\varepsilon$-homotopy provided that the track of each point has diameter $<\varepsilon$. A proper map $f: X \rightarrow Y$ is said to be an $\varepsilon$-equivalence if there is a map $g: Y \rightarrow X$ such that $f \circ g$ is $\varepsilon$-homotopic to id and $g \circ f$ is $f^{-1}(\varepsilon)$-homotopic to id. This latter statement means that there is a homotopy $\varphi_{t}: g \circ f \simeq \mathrm{id}$ such that $f \circ \varphi_{t}$ is an $\varepsilon$-homotopy. If $A \subset Y$ is closed, then the proper map $f: X \rightarrow Y$ is said to be an $\alpha$-equivalence over $A$ (or e-equivalence over $A$ ) if there is a map $g: A \rightarrow X$ such that $f \circ g$ is $\alpha$-homotopic (or $\varepsilon$-homotopic) to the inclusion $A \hookrightarrow Y$, and $g \circ f \mid f^{-1}(A)$ is $f^{-1}(\alpha)$-homotopic (or $f^{-1}(\varepsilon)$ homotopic) to $f^{-1}(A) \hookrightarrow X$. We call $g$ an $\alpha$-inverse of $f$ over $A$.

In general, "id" will be used to represent identity maps and "inc" will be used for inclusion maps. For any $X$ and $A \subset X, \AA$ denotes the (topological) interior of $A$ and $\operatorname{Bd}(A)$ denotes the boundary of $A$. If $X$ has a specified metric and $x \in X$, then $B_{\varepsilon}(x)$ 
is the open $\varepsilon$-ball around $x$. Also, $f \mid A: A \rightarrow Y$ is simply written $f \mid: A \rightarrow Y$.

A proper map $f: X \rightarrow Y$ is said to be a fine equivalence provided that it is an $\alpha$-equivalence, for all open covers $\alpha$ of $Y$. We say that $f: X \rightarrow Y$ is cell-like (or $\mathrm{CE}$ ) if $f$ is surjective and all point-inverses have trivial shape in the sense of Borsuk [2]. We recall the following basic connection between these two notions [16]:

A proper map $f: X \rightarrow Y$ between ANRs is a fine equivalence iff it is cell-like.

A proper map $f: X \rightarrow Y$ is said to be contractible provided that it is surjective and all point-inverses are contractible (in themselves). Thus the above result implies that any contractible map of ANRs is an $\alpha$-equivalence, for all open covers $\alpha$ of the range. In the following result we collect some basic facts about $\alpha$-equivalences which are easy consequences of the definitions involved.

Proposition 2.2. (i) If $f: X \rightarrow Y$ is an $\alpha$-equivalence and $f$ is $\beta$-homotopic to a proper map $\bar{f}: X \rightarrow Y$, then $\bar{f}$ is a $\operatorname{St}^{2}(\beta, \alpha)$ equivalence.

(ii) If $\bar{f}: Y \rightarrow Z$ is a $\beta$-equivalence and $f: X \rightarrow Y$ is an $\bar{f}^{-1}(\alpha)$ equivalence, for any open covers $\alpha, \beta$ of $Z$, then $\overline{f f}: X \rightarrow Z$ is a $\operatorname{St}^{2}(\beta, \alpha)$-equivalence.

Proof. (i) If $g: Y \rightarrow X$ is an $\alpha$-inverse of $f$, then it is easy to see that $g$ is a $\operatorname{St}^{2}(\beta, \alpha)$-inverse of $\bar{f}$.

(ii) Let $\alpha$ be any open cover of $Z$ and let $g: Y \rightarrow X$ be an $\bar{f}^{-1}(\alpha)$-inverse of $f$. Similarly let $\bar{g}: Z \rightarrow Y$ be a $\beta$-inverse of $\bar{f}$. We leave it as an easy exercise for the reader to check that $g \bar{g}$ : $Z \rightarrow X$ is a $\operatorname{St}^{2}(\beta, \alpha)$-inverse of $\tilde{f} f: X \rightarrow Z$.

REMARKS. There is a version of (ii) above in which $\bar{f}$ is only assumed to be a $\beta$-equivalence over $A \subset Z$. In this case (ii) asserts that if $f: X \rightarrow Y$ is an $\bar{f}^{-1}(\alpha)$-equivalence over $A$, then $\bar{f} f: X \rightarrow Z$ is a $\operatorname{St}^{2}(\beta$, $\alpha$ )-equivalence over $A$. Finally we remark that the result from [16] (quoted above), in conjunction with (ii), implies that if $f: X \rightarrow Y$ is a cell-like map of ANRs and $\bar{f}: Y \rightarrow Z$ is a $\beta$-equivalence, then $\bar{f} f$ : $X \rightarrow Z$ is also a $\beta$-equivalence.

By a polyhedron we will mean a space which admits a PL structure in the sense of [17]. We will use notions from [17] such as subpolyhedron, PL map, PL collapse, etc.

For any map $f: X \rightarrow Y$ we let $M(f)$ denote its mapping cylinder. 
It is the quotient space obtained from the disjoint union, $X \times[0,1] \Perp$ $Y$, by identifying $(x, 1)$ with $f(x)$. We write $M(f)=X \times[0,1) \cup Y$ and identify $X$ with its 0-level, $X \times\{0\} \subset M(f)$. By the rays of $M(f)$ we mean the intervals $\{x\} \times[0,1) \cup\{f(x)\} \subset M(f)$. There is a natural collapse to the base, $c: M(f) \rightarrow Y$, defined by $c \mid Y=$ id and $c(x, t)=f(x)$, for all $(x, t) \in X \times[0,1)$.

We will also need the direct mapping cylinder construction. Let $X$ be a space and $f: X \rightarrow X$ a map. The infinite direct mapping cylinder of $f$, denoted $D_{f}$, is the quotient space obtained from the disjoint union,

$$
\cdots \Perp X \times[-1,0] \Perp X \times[0,1] \Perp X \times[1,2] \Perp \cdots,
$$

by identifying $(x, n)$ in $X \times[n-1, n]$ with $(f(x), n)$ in $X \times$ $[n, n+1]$. Note that $D_{f}$ is just a union of countably many copies of $M(f)$. In a natural way $D_{f}$ may be set-wise identified with $X \times$ $R$. We use $D_{f}[a, b]$ to denote the subset of $D_{f}$ which corresponds to the subset $X \times[a, b]$ of $X \times R$.

A map $f: X \rightarrow Y$ is a homotopy domination if there is a map $g: Y \rightarrow X$ such that $f \circ g \simeq$ id. Let $\left(X, X_{0}\right)$ be a compact ANR pair, $X_{0} \neq \varnothing$, and let $e: X \rightarrow X$ be a homotopy idempotent rel $X_{0}$. This means that $e \mid X_{0}=\mathrm{id}$ and there exists a homotopy $e_{t}: e \simeq e^{2}$ rel $X_{0}$. Note that the subset of $D_{e}$ corresponding to $X_{0} \times R$ is actually homeomorphic to $X_{0} \times R$. So we identify it with $X_{0} \times R$. Define $s: D_{e} \rightarrow X$ by $s(x, t)=e_{t-n}(x)$, for $(x, t) \in D_{e}[n, n+1)$. Note that $s$ is continuous. Let $i$ : $X \rightarrow D_{e}$ be the map defined by $i(x)=(x, 0)$. We will need some information concerning this special situation which comes up in $\S 4$. Compare with [9].

Proposition 2.3. The composition $i \circ s: D_{e} \rightarrow D_{e}$ is a homotopy equivalence. Moreover, $i$ is a homotopy domination and we can choose a right inverse of $i, s^{\prime}: D_{e} \rightarrow X$, and a homotopy $h_{t}: i \circ s^{\prime} \simeq$ id such that $s^{\prime} \mid X_{0} \times R=$ proj: $X_{0} \times R \rightarrow X_{0}, \quad h_{t} \mid X_{0} \times R$ is given by $h_{t}(x, r)=(x, t r)$, and $s^{\prime} \circ i=e$.

Proof. Let $\alpha: D_{e} \rightarrow D_{e}$ be a map such that $\alpha \mid A=\mathrm{id}$, where $A=i(X)$, the subset of $D_{e}$ identified with $X \times\{0\}$. Also let $A_{0}=$ $X_{0} \times\{0\} \subset A$.

Assertion. We can choose a homotopy inverse of $\alpha$, say $\beta$ : $D_{e} \rightarrow D_{e}$, such that $\beta \mid A=\mathrm{id}, \beta \circ \alpha \simeq$ id rel $A, \alpha \circ \beta \simeq$ id rel $A$.

Proof. It suffices to prove that $\alpha$ induces isomorphisms on all homotopy groups, $\pi_{n}\left(D_{e}\right)$. If $j$ is the inclusion-induced homomorphism, $\pi_{n}(A) \stackrel{j}{\rightarrow} \pi_{n}\left(D_{e}\right)$, then the commutativity of 


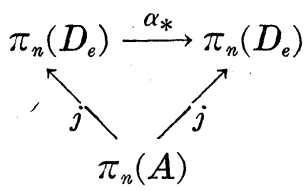

implies that all we have to do is prove that $j$ is surjective. To see this choose any element $[\varphi] \in \pi_{n}\left(D_{e}\right)$. By deforming down the rays of the mapping cylinders in $D_{e}$, and then using the fact that $e \simeq e^{2}$, we can easily find an element $[\psi] \in \pi_{n}(A)$ for which $j([\psi])=$ $[\varphi]$.

Returning to the proof of Proposition 2.3 consider $s: D_{e} \rightarrow X$ and note that $i \circ s \mid A$ is given by $i \circ s(x, 0)=(e(x), 0)$. We only have a homotopy in $D_{e}, i \circ s \mid A \simeq$ inc, obtained by deforming down the rays of $D_{e}[0,1]$, applying $e^{2} \simeq e$, and coming back up the rays of $D_{e}[0,1]$. Using Proposition 2.1 we can extend this homotopy $i \circ s \mid A \simeq$ id $\mid A$ to a homotopy $i \circ s \simeq \alpha$, where $\alpha \mid A=$ id. By the Assertion, $\alpha$ is a homotopy equivalence. Thus $i \circ s$ is a homotopy equivalence as we set out to prove.

Choose $\beta: D_{e} \rightarrow D_{e}$ as in the Assertion above and consider the homotopy

$$
h_{t}^{\prime}: i \circ s \circ \beta \simeq \alpha \circ \beta \simeq \mathrm{id},
$$

where the first homotopy comes from $i \circ s \simeq \alpha$, and the second comes from the Assertion. Thus $s^{\prime \prime}=s \circ \beta$ is a right inverse of $i$. We note that $s^{\prime \prime}(x, 0)=e(x)$, for $(x, 0) \in A$, and $h_{t}^{\prime}(x, 0)=(x, r)$, for all $x \in X_{0}$ (i.e., $h_{t}^{\prime}$ preserves the $X_{0}$-coordinate in $A_{0}$ ). We will now modify $s^{\prime \prime}$ and $h_{t}^{\prime}$ to get our desired $s^{\prime}$ and $h_{t}$.

Since $s^{\prime \prime}(x, 0)=x$, for all $x \in X_{0}$, we can find a homotopy of

$$
s^{\prime \prime} \mid A \cup\left(X_{0} \times R\right) \text { to } \mathrm{id}_{A} \cup \operatorname{proj} \mid X_{0} \times R,
$$

where each level of the homotopy agrees with $s^{\prime \prime}$ on $A$. By Proposition 2.1 we can extend this homotopy to a homotopy $s^{\prime \prime} \simeq s^{\prime}$, where $s^{\prime}\left|X_{0} \times R=\operatorname{proj}\right| X_{0} \times R$ and $s^{\prime}(x, 0)=s^{\prime \prime}(X, 0)=e(x)$, for $(x, 0) \in A$. We then get a homotopy

$$
h_{t}^{\prime \prime}: i \circ s^{\prime} \simeq i \circ s^{\prime \prime} \simeq \mathrm{id},
$$

where the first homotopy comes from $s^{\prime} \simeq s^{\prime \prime}$, and the second is $h_{t}^{\prime}$. Thus $h_{t}^{\prime \prime}(x, 0)=(x, r)$, for all $x \in X_{0}$ and $t \in[0,1]$. Our final step is to show how $h_{t}^{\prime \prime}$ can be modified to obtain our required $h_{t}$.

Let $H: D_{e} \times I \rightarrow D_{e}$ be defined by $H(z, t)=h_{t}^{\prime \prime}(z)$ and let

$$
S=\left(D_{e} \times\{0,1\}\right) \cup\left(X_{0} \times R \times I\right) \subset D_{e} \times I .
$$


The condition $h_{t}^{\prime \prime}(x, 0)=(x, r)$, for $x \in X_{0}$, permits us to find a homotopy $F_{u}: H \mid S \simeq F_{1}$, where $F_{u}\left|D_{e} \times\{0\}=i \circ s^{\prime}, F_{u}\right| D_{e} \times\{1\}=\mathrm{id}$, and $F_{1} \mid X_{0} \times R \times I$ is given by $F_{1}((x, r), t)=(x, t r)$. By Proposition 2.1 we can extend $F_{1}$ to a map $K: D_{e} \times I \rightarrow D_{e}$, and $h_{t}(z)=K(z, t)$ fulfills our requirements.

We will need one more result in $\S 4$. In addition to the above notation let $\left(Y, Y_{0}\right)$ be a compact ANR pair and let $u:\left(X, X_{0}\right) \rightarrow$ $\left(Y, Y_{0}\right), v:\left(Y, Y_{0}\right) \rightarrow\left(X, X_{0}\right)$ be maps such that $u \circ v\left|Y_{0}=\mathrm{id}, v \circ u\right| X_{0}=$ $\mathrm{id}, e=v \circ u$, and $u \circ v \simeq \mathrm{id} \operatorname{rel} Y_{0}$.

Proposition 2.4. The compositions $u \circ s, u \circ s^{\prime}: D_{e} \rightarrow X \rightarrow Y$ are homotopy equivalences. Moreover $u \circ s^{\prime}$ has a homotopy inverse $i \circ v$ : $Y \rightarrow X \rightarrow D_{e}$.

Proof. Here are the homotopies which show that $i \circ v$ is a homotopy inverse of $u \circ s^{\prime}$. It is equally easy to show that $u \circ s$ is a homotopy equivalence.

(1) $u \circ s^{\prime} \circ i \circ v=u \circ s \circ i \circ v=u \circ e \circ v=u \circ v \circ u \circ v \simeq \mathrm{id}$, where the homotopy comes from $u \circ v \simeq \mathrm{id}$.

(2) $i \circ v \circ u \circ s^{\prime}=i \circ e \circ s^{\prime} \simeq i \circ s^{\prime} \simeq \mathrm{id}$, where the first homotopy comes from $i_{\circ} e \simeq i$ (by deforming down the rays of $D_{e}[0,1]$ ), and the second is just the homotopy $h_{t}$ of Proposition 2.3.

REMARK. The statement that $u \circ s: D_{e} \rightarrow Y$ is a homotopy equivalence suffices for the proof of Theorem 4.3. However, in the Addendum to Theorem 4.3 we will need to exercise some more control, and for this we need the explicit construction of $u \circ s^{\prime}$ in the statement and proof given above.

Finally we introduce one more notational convention which will be commonplace in the sequel. Let $f, g: X \rightarrow Y$ be maps and let $A \subset Y$. We say that $f=g$ over $A$ if $f^{-1}(A)=g^{-1}(A)$ and $f\left|f^{-1}(A)=g\right| f^{-1}(A)$. In general we say that $f$ has property $P$ over $A$ if $f \mid f^{-1}(A): f^{-1}(A) \rightarrow$ $A$ has property $P$.

3. Preliminaries on equivalences. In this section we will establish some general results about $\alpha$-equivalences which will be needed in the sequel.

Proposition 3.1. Let $(X, Y)$ be a compact ANR pair with $i$ : $Y \hookrightarrow X$ an $\alpha$-equivalence, for any open cover $\alpha$ of $X$. Then there exists a map $g: X \rightarrow Y$ such that $g \mid Y=\mathrm{id}_{Y}$ and inc $\circ g$ is $\mathrm{St}^{4}(\alpha)$-homotopic to $\mathrm{id}_{X}$ rel $Y$. 
Proof. If reference to the cover $\alpha$ is omitted, then the result is well-known [22, p. 31]. Let $g_{1}: X \rightarrow Y$ be an $\alpha$-inverse of $i$. This means that we have $\alpha$-homotopies $g_{1} \simeq \mathrm{id}_{X}$ and $g \mid Y \simeq \mathrm{id}_{Y}$. By Proposition 2.1 there is an $\alpha$-homotopy $g_{1} \simeq g$ such that $g \mid Y=$ id. The $\alpha$-homotopies id $\simeq g_{1}$ and $g_{1} \simeq g$ combine to give us a St $(\alpha)$ homotopy id $\simeq g$. Call this $\operatorname{St}(\alpha)$-homotopy $F: X \times[0,1] \rightarrow X$, where $F_{0}=$ id and $F_{1}=g$.

Define a homotopy

$$
G:[(X \times\{0,1\}) \cup(Y \times[0,1])] \times[0,1] \longrightarrow X
$$

by the equations

$$
\begin{aligned}
& G((x, 0), t)=x, \text { for all } x \in X, \\
& G((x, 1), t)=F(g(x), 1-t), \text { for all } x \in X, \\
& G((x, s), t)=F(x,(1-t) s), \text { for all } x \in Y .
\end{aligned}
$$

Note that $G_{0}$ extends to $F: X \times[0,1] \rightarrow X$ and $G$ is a St $(\alpha)$-homotopy. Thus $G_{1}$ extends to $H: X \times I \rightarrow X$ which is a $\operatorname{St}^{4}(\alpha)$-homotopy of id to $g$ rel $Y$.

Proposition 3.2. Let $\left(X, X_{0}\right),\left(Y, Y_{0}\right)$ be compact ANR pairs and let $f: X \rightarrow Y$ be an $\alpha$-equivalence such that $f \mid X_{0}: X_{0} \rightarrow Y_{0}$ is a homeomorphism. Then there exists a map $g: Y \rightarrow X$ such that $g\left|Y_{0}=f^{-1}\right| Y_{0}$ and there are homotopies $f g \simeq \mathrm{id}$ rel $Y_{0}, g f \simeq \mathrm{id}$ rel $X_{0}$, where the former is a $\mathrm{St}^{4}(\alpha)$-homotopy and the latter is an $f^{-1}\left(\mathbf{S t}^{9}(\alpha)\right)$-homotopy.

Proof. Form the mapping cylinder $M(f)$ and let $\varphi: X \rightarrow[0,1]$ be a map for which $\varphi^{-1}(1)=X_{0}$. Define $Z \subset M(f)$ to be the union of the base $Y$ with all $(x, t) \in X \times[0,1)$ for which $\varphi(x) \leqq t<1$. Thus

$$
Z=Y \cup\left(\cup\left\{\{x\} \times[\varphi(x), 1) \mid x \in X-X_{0}\right\}\right) .
$$

We have an embedding $f_{1}: X \rightarrow Z$ given by $f_{1}(x)=f(x)$, for $x \in X_{0}$, and $f_{1}(x)=(x, \varphi(x))$, for $x \in X-X_{0} . \quad Z$ is called a reduced mapping cylinder with top $f_{1}(X)$ and base $Y$. There is a natural collapse to the base, $c: Z \rightarrow Y$, obtained by restricting the collapse of $M(f)$ to $Z$. $Z$ is an ANR because it is a retract of the ANR $M(f)$.

Since $f: X \rightarrow Y$ is an $\alpha$-equivalence, it easily follows that $f_{1}: X \rightarrow$ $Z$ is a $c^{-1}(\alpha)$-equivalence. By Proposition 3.1 there is a map $g_{1}: Z \rightarrow$ $f_{1}(X)$ such that $g_{1} \mid f_{1}(X)=\mathrm{id}$ and $g_{1} \simeq \mathrm{id} \operatorname{rel} f_{1}(X)$ via a $\operatorname{St}^{4} c^{-1}(\alpha)$ homotopy. Then the reader can easily check that $g=f_{1}^{-1} g_{1} \mid Y: Y \rightarrow$ $X$ is our desired map. 
For our next result let $X, Y$ be polyhedra and $f: X \rightarrow Y$ a proper map. Let $c: M(f) \rightarrow Y$ denote the collapse of the mapping cylinder to its base.

Proposition 3.3. Let $\varphi: Y \rightarrow[0,4]$ be a map and let $\alpha$ be an open cover of $Y$ such that $\operatorname{diam} \varphi(U)<1 / 2$, for all $U \in \alpha$. If $f: X \rightarrow Y$ is an $\alpha$-equivalence over $\varphi^{-1}([0,3])$, then there is a map $\widetilde{g}: c^{-1} \Phi^{-1}([0,2]) \rightarrow X$ such that inc $\circ \widetilde{g}$ is $c^{-1} \mathrm{St}^{4}(\alpha)$-homotopic to id rel $f^{-1} \varphi^{-1}([0,2])$, with the homotopy taking place in $M(f)$.

Proof. For each $t \in[0,4]$ let $Y_{t}=\phi^{-1}([0, t])$ and choose a map $g: Y_{3} \rightarrow X$ which is an $\alpha$-inverse of $f$ over $Y_{3}$. Define a map $g_{1}: c^{-1}\left(Y_{3}\right) \rightarrow X$ by $g_{1}=g \circ c \mid c^{-1}\left(Y_{3}\right)$ and note that $g_{1} \mid f^{-1}\left(Y_{3}\right)$ is $f^{-1}(\alpha)$ homotopic to id. We will show how to perform two modifications of $g_{1}$ to arrive at our desired $\widetilde{g}: c^{-1}\left(Y_{2}\right) \rightarrow X$.

Using Proposition 2.1 we see that $g_{1}$ is $f^{-1}(\alpha)$-homotopic to a map $g_{2}: c^{-1}\left(Y_{3}\right) \rightarrow X$ such that $g_{2} \mid f^{-1}\left(Y_{3}\right)=$ id. We have a $c^{-1}(\alpha)$ homotopy

$$
g_{1} \simeq f \circ g_{1}=f \circ g \circ c\left|c^{-1}\left(Y_{3}\right) \simeq c\right| c^{-1}\left(Y_{3}\right) \simeq \mathrm{id},
$$

where the first homotopy comes from deforming down the rays of $M(f)$, the second comes from $f \circ g \simeq \mathrm{id}$, and the third comes from deforming back up the rays of $M(f)$. Thus we have a $c^{-1} \operatorname{St}(\alpha)$ homotopy $F: c^{-1}\left(Y_{3}\right) \times I \rightarrow M(f)$ from id to $g_{2}$. We define our required $\widetilde{g}: c^{-1}\left(Y_{2}\right) \rightarrow X$ by $\widetilde{g}=g_{2} \mid c^{-1}\left(Y_{2}\right)$. In analogy with [22, p. 31] we now show how to modify $F$ to obtain a $c^{-1} \mathrm{St}^{4}(\alpha)$-homotopy of $\widetilde{g}$ to id rel $f^{-1}\left(Y_{2}\right)$.

Define

$$
G:\left[\left(c^{-1}\left(Y_{2}\right) \times\{0,1\}\right) \cup f^{-1}\left(Y_{2} \times I\right)\right] \times I \longrightarrow M(f)
$$

by the equations

$$
\begin{aligned}
& G((x, 0), t)=x, \quad \text { for all } x \in c^{-1}\left(Y_{2}\right), \\
& G((x, 1), t)=F\left(g_{2}(x), 1-t\right), \quad \text { for all } x \in c^{-1}\left(Y_{2}\right), \\
& G((x, s), t)=F(x,(1-t) s), \quad \text { for all } x \in f^{-1}\left(Y_{2}\right) .
\end{aligned}
$$

Observe that in order for the third equation to make sense we must have $g_{2} \circ c^{-1}\left(Y_{2}\right) \subset c^{-1}\left(Y_{3}\right)$. This is the reason for choosing $\alpha$ in the prescribed manner.

Note that $G_{0}$ can be extended to $F \mid c^{-1}\left(Y_{2}\right) \times I$ and $G$ is a $c^{-1} \operatorname{St}(\alpha)$-homotopy. By Proposition 2.1 we can extend $G_{1}$ to a map $H: c^{-1}\left(Y_{2}\right) \times I \rightarrow M(f)$ which is $c^{-1} \operatorname{St}(\alpha)$-homotopic to $F \mid c^{-1}\left(Y_{2}\right) \times I$, thus implying that $H$ is a $c^{-1} \mathrm{St}^{4}(\alpha)$-homotopy. Then $H$ is our required $c^{-1} \mathrm{St}^{4}(\alpha)$-homotopy of id to $g_{2} \mid c^{-1}\left(Y_{2}\right) \operatorname{rel} f^{-1}\left(Y_{2}\right)$. 
Proposition 3.4. There exists a number $\varepsilon>0$ so that if $f: X \rightarrow$ $Y$ is a proper map of polyhedra, $\varphi: Y \rightarrow[-4,4]$ is a map, $\alpha$ is an open cover of $Y$ for which diam $\varphi(U)<\varepsilon$, for all $U \in \alpha$, and $f$ is an $\alpha$-equivalence over $\varphi^{-1}([-3,1])$ and $\varphi^{-1}([-1,3])$, then $f$ is a $\operatorname{St}^{19}(\alpha)$-equivalence over $\varphi^{-1}([-2,2])$.

Proof. Let $M(f)$ be the mapping cylinder of $f$. By Proposition 3.3 there is a map $g_{1}: c^{-1} \varphi^{-1}([-2.5, .5]) \rightarrow X$ for which inc $\circ g_{1}$ is $c^{-1} \mathrm{St}^{4}(\alpha)$-homotopic to id rel $f^{-1} \varphi^{-1}([-2.5, .5])$. Similarly there is a map $g_{2}: c^{-1} \varphi^{-1}([-.5,2.5]) \rightarrow X$ for which inc $\circ g_{2}$ is $c^{-1} \operatorname{St}^{4}(\alpha)$-homotopic to id rel $f^{-1} \phi^{-1}([-.5,2.5])$. The map $g_{1}$, along with the homotopy inc $\circ g_{1} \simeq$ id, easily give us a map $\widetilde{g}_{1}: M(f) \rightarrow M(f)$ such that $\widetilde{g}_{1}=g_{1}$ on $c^{-1} \varphi^{-1}([-2.4, .4]), \widetilde{g}_{1}=$ id on $X$, and $\widetilde{g}_{1} \simeq$ id rel $X$ via a $\operatorname{St}^{4} c^{-1}(\alpha)$ homotopy. Similarly there is a map $\widetilde{g}_{2}: M(f) \rightarrow M(f)$ such that $\widetilde{g}_{2}=g_{2}$ on $c^{-1} \mathscr{P}^{-1}([-.4,2.4]), \widetilde{g}_{2}=\mathrm{id}$ on $X$, and $\widetilde{g}_{2} \simeq$ id rel $X$ via a $\mathrm{St}^{4} c^{-1}(\alpha)$-homotopy.

Now define $g: c^{-1} \varphi^{-1}([-2,2]) \rightarrow X$ by $g=\widetilde{g}_{2} \circ \widetilde{g}_{1} \mid c^{-1} \varphi^{-1}([-2,2])$. (This makes sense if $\varepsilon$ is small enough.) Then inc $\circ g=\widetilde{g}_{2} \circ \widetilde{g}_{1}\left|\simeq \widetilde{g}_{1}\right| \simeq$ id, where these are both $\mathrm{St}^{4} c^{-1}(\alpha)$-homotopies. Therefore inc $\circ g \simeq$ id rel $f^{-1} \varphi^{-1}([-2,2])$ via a $\mathrm{St}^{9} c^{-1}(\alpha)$-homotopy.

Now define $\bar{g}: \varphi^{-1}([-2,2]) \rightarrow X$ by $\bar{g}=g \mid \phi^{-1}([-2,2])$. We leave it for the reader to check that $\bar{g}$ is a $\operatorname{St}^{19}(\alpha)$-inverse of $f$ over $\varphi^{-1}([-2,2])$. (Compare this with the checking needed in Proposition 3.2.)

4. A finiteness result. In this section we prove Theorem 4.3, a result which will only be needed in the next section. Its proof uses some material from Wall's finiteness obstruction theory which we summarize below in Theorems 4.1 and 4.2.

The statements of the following results require the reduced projective class group functor $\widetilde{K}_{0}$. Here is a brief description of just what we will need.

1. For every topological space $X$ there is an abelian group $\widetilde{K}_{0}(X)$. We will not need a definition of $\widetilde{K}_{0}(X)$, but for the interested reader it is the direct sum of all $\widetilde{K}_{0} Z\left[\pi_{1}(C)\right]$, where $C$ is a path component of $X$ and $Z\left[\pi_{1}(C)\right]$ denotes the integral group ring. (See $\left[23\right.$, p. 64] for a definition of $\widetilde{K}_{0} Z\left[\pi_{1}(C)\right]$.)

2. For each map $f: X \rightarrow Y$ there is induced a homomorphism $f_{*}: \widetilde{K}_{0}(X) \rightarrow \widetilde{K}_{0}(Y)$ so that $\widetilde{K}_{0}$ becomes a covariant functor from the homotopy category of topological spaces (and homotopy classes of maps) to the category of abelian groups (and homomorphisms).

3. It follows from the fundamental theorem of algebraic $K$-theory $[1, \mathrm{p} .663]$ that $\widetilde{K}_{0}(X)$ and $\mathrm{Wh}(X)$ are direct summands of $\mathrm{Wh}\left(X \times S^{1}\right)$, where Wh is the Whitehead group functor [11, p. 39]. Although this fact will not be needed in this section, it will be used in $\S 5$. 
Here is the basic geometric problem in which $\widetilde{K}_{0}$ is used. Let $X$ be a compact polyhedron, $Y$ be any ANR, and let $f: X \rightarrow Y$ be a homotopy domination (cf. $\S 2$ for a definition). In [23] Wall analyzed the problem of extending $f$ to a homotopy equivalence $\tilde{f}: \widetilde{X} \rightarrow Y$, where $\widetilde{X}$ is a compact polyhedron containing $X$ as a subpolyhedron. Here is the main result from [23] which solves this problem.

TheOREM 4.1. $f: X \rightarrow Y$ can be extended to a homotopy equivalence $\tilde{f}: \widetilde{X} \rightarrow Y$ (in the above manner) iff an obstruction $\sigma(Y)$ in $\widetilde{K}_{0}(Y)$ vanishes. $\sigma(Y)$ is independent of the choice of $f$ and $X$.

The main use of this result is the case in which $\pi_{1}(C)$ is free or free abelian, for each path component $C$ of $Y$; for then $\widetilde{K}_{0}(Y)=$ 0 and therefore $f$ extends in the required manner. (See $[23$, p. 67] for references.)

We now introduce some notation for the next result. Let an ANR $Y$ be written as the union of closed ANRs $Y_{1}$ and $Y_{2}$ with $Y_{0}=Y_{1} \cap Y_{2}$ also an ANR. Let $j_{i}$ be the inclusion $Y_{i} \hookrightarrow Y$, which induces a homomorphism $\left(j_{i}\right)_{*}: \widetilde{K}_{0}\left(Y_{i}\right) \rightarrow \widetilde{K}_{0}(Y)$. The following Sum Theorem computes $\sigma(Y)$ in terms of the $\sigma\left(Y_{i}\right)[19$, p. 48].

THEOREM 4.2. If each $Y_{i}$ is homotopically dominated by a compact polyhedron, then so is $Y$ and

$$
\sigma(Y)=\left(j_{1}\right)_{*} \sigma\left(Y_{1}\right)+\left(j_{2}\right)_{*} \sigma\left(Y_{2}\right)-\left(j_{0}\right)_{*} \sigma\left(Y_{\bullet}\right) \text {. }
$$

The main result. We now introduce some notation for Theorem 4.3 , the main result of this section. Consider a compact polyhedral pair $\left(Y_{0}, L\right)$, where $\tilde{K}_{0}(L)=0$. Form the polyhedron $Y=Y_{0} \cup(L \times$ $[0,6])$ by sewing $L \times[0,6]$ to $Y_{0}$ along $L \equiv L \times\{0\}$. For each $t$ let $Y_{t}=Y_{0} \cup(L \times[0, t])$ and let $\varphi: Y \rightarrow[0,6]$ be the map for which $\phi^{-1}([0, t])=Y_{t}$, for each $t$.

THEOREM 4.3. There exists an $\varepsilon>0$ such that if $X$ is any compact polyhedron and $f: X \rightarrow Y$ is any $\phi^{-1}(\varepsilon)$-equivalence over $Y_{5}$, then we can extend $X$ to a compact polyhedron $\widetilde{X}$ and define a map $\tilde{f}: \widetilde{X} \rightarrow Y$ such that

(1) $\tilde{f}$ is a homotopy equivalence,

(2) $\tilde{f}(\tilde{X}-X) \subset L \times[1,6]$,

(3) $\tilde{f}=f$ over $Y_{1}$.

Proof. We will use the direct mapping cylinder construction of $\S 2$ to reduce this problem to one in which Theorems 4.1 and 4.2 are applicable. Adopting the notation of Proposition 3.1 we consider the mapping cylinder $M(f)$, where $X \subset M(f)$ is the top and $Y \subset$ $M(f)$ is the base. If $c: M(f) \rightarrow Y$ is the collapse to the base, it 
follows from Proposition 3.1 that there is a map $g: c^{-1}\left(Y_{4}\right) \rightarrow X$ which is $c^{-1} \phi^{-1}(9 \varepsilon)$-homotopic to id rel $f^{-1}\left(Y_{4}\right)$. Consider the following compact subset of $c^{-1}\left(Y_{4}\right)$ :

$$
A=c^{-1}\left(Y_{3}\right) \cup Y_{4} \cup\left(\cup\left\{\{x\} \times[\varphi \circ f(x)-3,1) \mid x \in f^{-1}\left(\dot{Y}_{4}-\dot{Y}_{3}\right)\right\}\right) .
$$

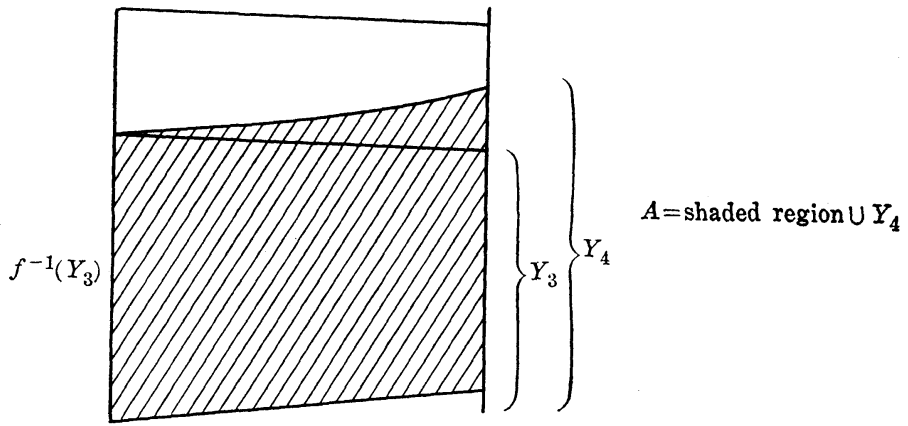

There is a natural retraction $r: M(f) \rightarrow A$ obtained by first retracting $M(f)$ to $A \cup Y$ by retracting down the rays of $M(f)$, and then retracting $Y$ to $Y_{4}$ by retracting down the rays of $L \times[0,6]$.

The map $X \hookrightarrow M(f) \stackrel{r}{\rightarrow} A$ is a homotopy domination with right inverse $A \stackrel{g}{\rightarrow} X$. Thus we get a homotopy idempotent $e: X \rightarrow X$ rel $f^{-1}\left(Y_{3}\right)$,

$$
e: X \subset M(f) \stackrel{r}{\longrightarrow} A \stackrel{g}{\longrightarrow} X .
$$

That is, $e \simeq e^{2}$ rel $f^{-1}\left(Y_{3}\right)$. Note that for a sufficiently small choice of $\varepsilon$, the homotopy $e \simeq e^{2}$ takes $f^{-1}(L \times[3,6])$ into $f^{-1}(L \times[2,6])$ at each level. Thus the restriction $e \mid f^{-1}(L \times[t, 6])$ is a homotopy idempotent of $f^{-1}(L \times[t, 6])$, for $0 \leqq t \leqq 2$. In what follows we will need the maps $D_{e} \underset{i}{\stackrel{s}{\rightleftarrows}} X$ which were described in $\S 2$ preceding

Proposition 2.3.

Note that the composition $f s: D_{e} \rightarrow Y$ is homotopic to the composition

$$
D_{e} \stackrel{s}{\longrightarrow} X \subset M(f) \stackrel{r}{\longrightarrow} A \stackrel{c !}{\longrightarrow} Y \text {. }
$$

By Proposition 2.4, $D_{e} \stackrel{s}{\rightarrow} X \hookrightarrow M(f) \stackrel{r}{\rightarrow} A$ is a homotopy equivalence. Since $c \mid: A \rightarrow Y$ is clearly a homotopy equivalence we conclude that $f s: D_{e} \rightarrow Y$ is a homotopy equivalence. Our strategy is to define $\widetilde{f}=(f s) \tilde{i}$, where $\tilde{i}: \widetilde{X} \rightarrow D_{e}$ is an extension of $i: X \rightarrow D_{e}$ to a homotopy equivalence. In order to extend $i$ to such a homotopy equivalence we will have to invoke the condition $\widetilde{K}_{0}(L)=0$.

Now choose compact subpolyhedra $K_{1}$ and $K_{2}$ of $X$ so that

$$
\begin{aligned}
f^{-1}(L \times[2,6]) & \subset K_{1} \subset f^{-1}(L \times[5 / 3,6]) \subset f^{-1}(L \times[4 / 3,6]) \subset K_{2} \\
& \subset f^{-1}(L \times[1,6]) .
\end{aligned}
$$


Let $e_{j}$ denote the restriction of $e$ to $K_{j}, j=1,2$. By restriction the homotopy domination $i: X \rightarrow D_{e}$ gives us homotopy dominations $i_{j}: K_{j} \rightarrow D_{e_{j}}$.

Assertion. $i_{2}: K_{2} \rightarrow D_{e_{2}}$ can be extended to a homotopy equivalence (in the manner of Theorem 4.1).

Proof. Using Theorem 4.1 it suffices to prove that the obstruction $\sigma\left(D_{e_{2}}\right)$ is zero. To do this first decompose $D_{e_{2}}$ as $D_{e_{2}}=D_{e_{1}} \cup$ $\left(D_{e_{2}}-\dot{D}_{e_{1}}\right)$, and note that $D_{e_{1}}, D_{e_{2}}-\dot{D}_{e_{1}}$, and $D_{e_{1}} \cap\left(D_{e_{2}}-\dot{D}_{e_{1}}\right)$ are all homotopically dominated by compact polyhedra. In fact, $D_{e_{1}}$ is dominated by $K_{1}, D_{e_{2}}-{\stackrel{\circ}{e_{1}}}_{e_{1}}=\left(K_{2}-\grave{K}_{1}\right) \times R$, and $D_{e_{1}} \cap\left(D_{e_{2}}-\dot{D}_{e_{1}}\right)=$ $\mathrm{Bd}\left(K_{1}\right) \times R$. Using Theorem 4.2 we have

$$
\sigma\left(D_{e_{2}}\right)=\sigma\left(D_{e_{1}}\right)+\sigma\left(D_{e_{2}}-{\stackrel{\circ}{e_{1}}}_{1}\right)-\sigma\left(D_{e_{1}} \cap\left(D_{e_{2}}-{\stackrel{\circ}{D_{1}}}_{e_{1}}\right)\right),
$$

where we have omitted writing down the obvious inclusion-induced homomorphisms on the right hand side. Since $D_{e_{2}}-\stackrel{\circ}{D}_{e_{1}}=\left(K_{2}-\stackrel{\circ}{K}_{1}\right) \times$ $R$ and $D_{e_{1}} \cap\left(D_{e_{2}}-\dot{D}_{e_{1}}\right)=\operatorname{Bd}\left(K_{1}\right) \times R$ we observe that the latter two terms on the right hand side vanish. Thus $\sigma\left(D_{e_{2}}\right)$ is an element of the inclusion-induced image of $\widetilde{K}_{0}\left(D_{e_{1}}\right)$ in $\widetilde{K}_{0}\left(D_{e_{2}}\right)$.

To finish the proof of our Assertion it suffices to prove that the inclusion-induced image of $\widetilde{K}_{0}\left(D_{e_{1}}\right)$ in $\widetilde{K}_{0}\left(D_{e_{2}}\right)$ is zero. Let $s_{1}$ : $D_{e_{1}} \rightarrow K_{1}$ be the restriction of $s: D_{e} \rightarrow X$ to $D_{e_{1}}$, and note that Proposition 2.2 implies that the composition

$$
\theta: D_{e_{1}} \stackrel{s_{1}}{\longrightarrow} K_{1} \stackrel{e_{1}}{\longrightarrow} K_{1} \stackrel{i_{1}}{\longrightarrow} D_{e_{1}}
$$

is a homotopy equivalence (because $i_{1} \circ e_{1}$ is homotopic to $i_{1}$ ). Therefore $\theta$ induces an isomorphism $\theta_{*}: \widetilde{K}_{0}\left(D_{e_{1}}\right) \rightarrow \widetilde{K}_{0}\left(D_{e_{1}}\right)$. So it suffices to prove that the composition

$$
\theta^{\prime}: D_{e_{1}} \stackrel{\theta}{\longrightarrow} D_{e_{1}} \longrightarrow D_{e_{2}}
$$

induces the 0-map from $\widetilde{K}_{0}\left(D_{e_{1}}\right)$ to $\widetilde{K}_{0}\left(D_{e_{2}}\right)$. Clearly $\theta^{\prime}$ is equivalent to the composition

$$
\begin{aligned}
& D_{e_{1}} \stackrel{s_{1}}{\longrightarrow} K_{1} \hookrightarrow f^{-1}(L \times[5 / 3,6]) \stackrel{r}{\longrightarrow} A \cap c^{-1}(L \times[5 / 3,6]) \\
& \stackrel{g}{\longrightarrow} f^{-1}(L \times[4 / 3,6]) \smile K_{2} \stackrel{i_{2}}{\longrightarrow} D_{e_{2}} .
\end{aligned}
$$

Applying the functor $\widetilde{K}_{0}$ we conclude from this that $\left(\theta^{\prime}\right)_{*}: \widetilde{K}_{0}\left(D_{e_{1}}\right) \rightarrow$ $\widetilde{K}_{0}\left(D_{e_{2}}\right)$ factors through $\widetilde{K}_{0}\left(A \cap c^{-1}(L \times[5 / 3,6])\right)$. But $A \cap c^{-1}(L \times$ $[5 / 3,6])$ is homotopy equivalent to $L$. Thus $\widetilde{K}_{0}\left(A \cap c^{-1}(L \times[5 / 3,6])\right)=0$, which implies that $\left(\theta^{\prime}\right)_{*}\left(\widetilde{K}_{0}\left(D_{e_{1}}\right)\right)=0$.

Using the above Assertion we can extend $i_{2}: K_{2} \rightarrow D_{e_{2}}$ to a homotopy equivalence $\widetilde{i}_{2}: \widetilde{K}_{2} \rightarrow D_{e_{2}}$, where $\widetilde{K}_{2}$ is a compact polyhedron 
containing $K_{2}$ as a subpolyhedron. This implies that $i: X \rightarrow D_{e}$ extends to $\tilde{i}: \tilde{X} \rightarrow D_{e}$ by defining $\widetilde{X}=X \cup \widetilde{K}_{2}$ (sewn together along $K_{2}$ ) and setting $\tilde{i}=\tilde{i}_{2}$ on $\widetilde{K}_{2}$. It is easy to prove that $\tilde{i}$ is itself a homotopy equivalence because $D_{e}-\stackrel{\circ}{D}_{e_{2}}=\left(X-\stackrel{\circ}{K}_{2}\right) \times R$. Define $\tilde{f}$ : $\widetilde{X} \rightarrow Y$ to be the following composition:

$$
\tilde{f}: \tilde{X} \stackrel{i}{\longrightarrow} D_{e} \stackrel{s}{\longrightarrow} X \stackrel{f}{\longrightarrow} Y \text {. }
$$

We know from Proposition 2.4 that $D_{e} \stackrel{s}{\rightarrow} X \hookrightarrow M(f) \stackrel{r}{\rightarrow} A$ is a homotopy equivalence, and this easily implies that $D_{e} \stackrel{s}{\rightarrow} X \stackrel{f}{\rightarrow} Y$ is a homotopy equivalence. Thus $\widetilde{f}: \widetilde{X} \rightarrow Y$ is a homotopy equivalence and it is clear that $\tilde{f}=f$ over $Y_{1}$.

Finally, in the following Addendum we improve the above result so that a certain homotopy inverse of $\tilde{f}$ is constructed subject to restrictions. For additional notation let $\alpha$ be an open cover of $Y$ and assume that $f: X \rightarrow Y$ is also an $\alpha$-equivalence over $Y_{5}$.

Addendum to Theorem 4.3. We can choose the homotopy equivalence $\tilde{f}: \widetilde{X} \rightarrow Y$ so that in addition to satisfying (1)-(3) of the statement of Theorem 4.3, it has a homotopy inverse, $\widetilde{g}: Y \rightarrow \widetilde{X}$, and homotopies $\theta_{t}: \widetilde{f} \circ \widetilde{g} \simeq \mathrm{id}, \varphi_{t}: \widetilde{g} \circ \widetilde{f} \simeq \mathrm{id}$ which satisfy the following properties:

(1) $\theta_{t}$ is a $\mathrm{St}^{9}(\alpha)$-homotopy on $Y_{1}$, and on $Y-\stackrel{\circ}{1}_{1}$ it takes place in $Y-\dot{Y}_{0}$.

(2) $\varphi_{t}$ is a $f^{-1} \mathrm{St}^{4}(\alpha)$-homotopy on $f^{-1}\left(Y_{1}\right)$, and on $\tilde{f}^{-1}\left(Y-\dot{Y}_{1}\right)$ it takes place in $\widetilde{f}^{-1}\left(Y-\dot{Y}_{0}\right)$.

Proof. We will redefine $\tilde{f}$ slightly so that we can write down a homotopy inverse $\widetilde{g}$ in terms of the control given in Proposition 2.4. Using the notation set up in the proof of Theorem 4.3 we know that $e: X \rightarrow X$ is a homotopy idempotent $\operatorname{rel}\left(X-\stackrel{\circ}{K}_{1}\right)$. If $s^{\prime}: D_{e} \rightarrow X$ is defined as in Proposition 2.3, then $s^{\prime} \mid\left(X-\stackrel{\circ}{K}_{1}\right) \times R=$ proj: $(X-$ $\left.\stackrel{\circ}{K}_{1}\right) \times R \rightarrow X-\dot{K}_{1}$. By Proposition 2.4 we know that $D_{e} \stackrel{s^{\prime}}{\rightarrow} X \hookrightarrow$ $M(f) \stackrel{r}{\rightarrow} A$ is a homotopy equivalence with homotopy inverse $A \stackrel{g}{\rightarrow}$ $X \stackrel{i}{\rightarrow} D_{e}$, where $g: c^{-1}\left(Y_{4}\right) \rightarrow X$ is chosen so that it is $c^{-1} \mathrm{St}^{4}(\alpha)$-homotopic to id rel $f^{-1}\left(Y_{4}\right)$. Moreover, by Proposition 2.4 we can choose homotopies $r \circ s^{\prime} \circ i \circ g \simeq$ id and $i \circ g \circ r \circ s^{\prime} \simeq$ id subject to the following restrictions:

(1) $r \circ s^{\prime} \circ i \circ g \simeq$ id via a homotopy (in $A$ ) which is a $c^{-1} \operatorname{St}^{9}(\alpha)$ homotopy on $c^{-1}\left(Y_{1}\right)$, and on $A-c^{-1}\left(\dot{Y}_{1}\right)$ it takes place in $A-c^{-1}\left(\dot{Y}_{0}\right)$.

(2) $i \circ g \circ r \circ s^{\prime} \simeq$ id via a homotopy (in $D_{e}$ ) which takes $D_{e_{1}}$ into $D_{e_{1}}$ and on $D_{e}-\dot{D}_{e_{1}}=\left(X-\dot{K}_{1}\right) \times R$ it preserves the $\left(X-\dot{K}_{1}\right)$-coordinate. 
Now define $\tilde{f}: \tilde{X} \rightarrow Y$ to be the following composition:

$$
\widetilde{f}: \tilde{X} \stackrel{\tilde{i}}{\longrightarrow} D_{e} \stackrel{s^{\prime}}{\longrightarrow} X \stackrel{f}{\longrightarrow} Y .
$$

It is clear that $\tilde{f}$ satisfies properties (1)-(3) of the statement of Theorem 4.3.

Using the fact that $D_{e}-{\stackrel{\circ}{D_{2}}}_{\tilde{q}_{2}}=\left(X-\dot{K}_{2}\right) \times R$ it is easy to construct a homotopy inverse of $\tilde{i}, j: D_{e} \rightarrow \widetilde{X}$, so that $j: \mid\left(X-\stackrel{\circ}{K}_{2}\right) \times$ $R=$ proj: $\left(X-\dot{K}_{2}\right) \times R \rightarrow X-\dot{K}_{2}$ and so that we have homotopies $\tilde{i} \circ j \simeq \mathrm{id}, j \circ \tilde{i} \simeq \mathrm{id}$ subject to the following restrictions:

(1) $\tilde{i} \circ j \simeq$ id via a homotopy which takes $D_{e_{2}}$ into $D_{e_{2}}$, and on $\left(X-\dot{K}_{2}\right) \times R$ it preserves the $\left(X-\dot{K}_{2}\right)$-coordinate.

(2) $j \circ \tilde{i} \simeq$ id via a homotopy which is the identity on $X-\dot{K}_{2}$, and on $K_{2}$ it takes place in $K_{2}$. (Indeed, $j$ can be taken to be $s^{\prime}$ : $D_{e} \rightarrow X \hookrightarrow \widetilde{X}$, for $s^{\prime}$ is a right inverse of $i$ and $\tilde{i}$ extends $i$ to a homotopy equivalence.)

Then $\tilde{f}: \widetilde{X} \rightarrow Y$ has a homotopy inverse,

$$
\tilde{g}: Y \subset M(f) \stackrel{r}{\longrightarrow} A \stackrel{g}{\longrightarrow} X \stackrel{i}{\longrightarrow} D_{e} \stackrel{j}{\longrightarrow} \tilde{X} .
$$

We leave it to the reader to check that $\widetilde{g}$ fulfills our requirements.

5. The handle lemma. In this section we use Theorem 4.3 to prove the handle lemma, which is the main technical step of this paper. It is essentially an "extension theorem" for $\varepsilon$-equivalences. The proof uses torus geometry in the customary manner (cf. [6] and [21]). For notation, let $B_{r}^{n}$ denote the standard $n$-ball in Euclidean $n$-space $R^{n}$. Throughout this section $K$ will denote a compact polyhedron such that $\mathrm{Wh}\left(K \times T^{n}\right)=0$, for all $n \geqq 0$. Also $p: Z \times K \rightarrow Z$ will always denote projection to $Z$, for any space $Z$.

Handle Lemma. For each $\varepsilon>0$ there exists $a \delta>0$ so that if $X$ is a polyhedron and $f: X \rightarrow R^{n} \times K$ is a proper map which is a $p^{-1}(\delta)$-equivalence over $B_{3}^{n} \times K$, then there exists a polyhedron $\widetilde{X}, a$ proper map $\tilde{f}: \tilde{X} \rightarrow R^{n} \times K$, and a PL homeomorphism $\varphi: \tilde{f}^{-1}\left(\dot{B}_{1}^{n} \times\right.$ $K) \rightarrow f^{-1}\left(\dot{B}_{1}^{n} \times K\right)$ such that

(1) $\tilde{f}$ is a $p^{-1}(\varepsilon)$-equivalence,

(2) $\widetilde{f}$ is a PL homeomorphism over $\left(R^{n}-B_{5}^{n}\right) \times K$,

(3) $f \varphi=\widetilde{f}$ over $\stackrel{\circ}{B}_{1}^{n} \times K$.

REMARK. $\delta$ is independent of $K$.

Proof. For convenience let $B_{r}^{n}=[-r, r]^{n} \subset R^{n}$ and omit the subscript when $r=1$. We will use the metric on $R^{n}$ defined by 


$$
d\left(\left(x_{i}\right),\left(y_{i}\right)\right)=\max \left\{\left|x_{i}-y_{i}\right|\right\}_{i=1}^{n} .
$$

Let $e: R \rightarrow S^{1}$ be defined by $e(x)=\exp (\pi i x / 4)$, where $S^{1}$ is the set of complex numbers of absolute value 1 . Note that $e$ is a covering map, and if $T^{n}=S^{1} \times \cdots \times S^{1}$ is the $n$-torus, then $e^{n}=e \times \cdots \times$ $e: R^{n} \rightarrow T^{n}$ is also a covering map. Represent the punctured torus by $T_{0}^{n}=T^{n}-\left\{x_{0}\right\}$, where $x_{0} \notin e^{n}\left(B_{2}^{n}\right)$.

There are three large steps in the proof.
A. Pulling back to $T_{0}^{n} \times K$
B. Capping off to get $T^{n} \times K$
C. Lifting to $R^{n} \times K$.

There are also intermediate steps which are displayed in the diagram below. We remark here that steps $\mathrm{A}$ and $\mathrm{C}$ are easy in comparison with B. B requires Theorem 4.3 along with some results from simple homotopy theory. We assume that the reader is familiar with some of the standard results from simple homotopy theory such as those found in [11]. We will not require any infinite simple homotopy theory in this section.

Here is our "main diagram."

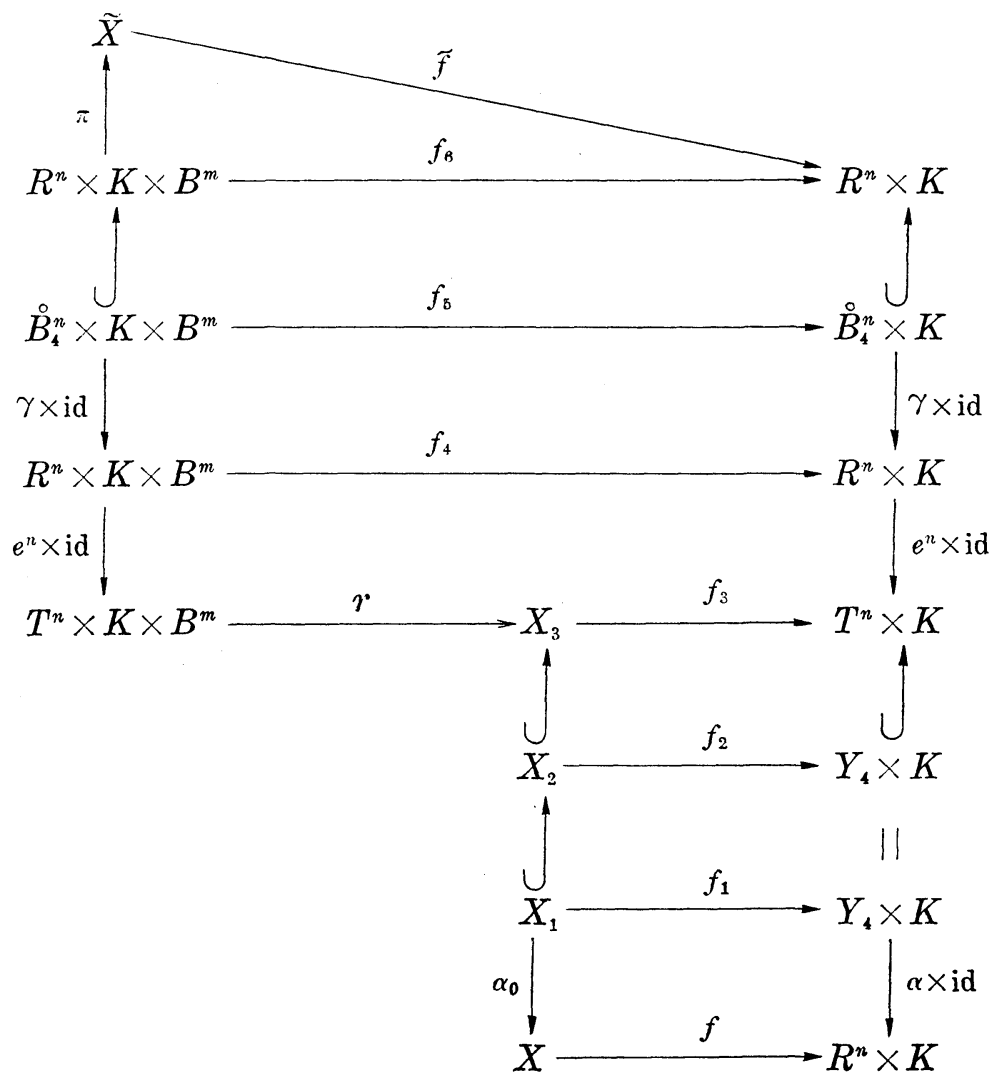


The goal of step $\mathrm{A}$ is the construction of the $\operatorname{map} f_{1}$, while the goal of $B$ is the construction of the maps $r$ and $f_{3}$. Finally, in step C we produce our desired "extension" $\tilde{f}$ at the top of the diagram.

A. Pulling back to $T_{0}^{n} \times K$. We will first need an immersion $\alpha: T_{0}^{n} \rightarrow R^{n}$. (An immersion is a local open embedding.) For the construction of $\alpha$ we refer the reader to [14] for an elementary proof. We may clearly assume that $\alpha\left(T_{0}^{n}\right) \subset B_{3}^{n}$, and by using the Schoenflies theorem we can adjust $\alpha$ to obtain the additional restriction, $\alpha \circ e^{n} \mid B_{2}^{n}=$ id (see [18, p. 48]). In what follows we assume that $n \geqq 2$. The case $n=1$ is much simpler (it does not require torus geometry).

Form the pull-back diagram,

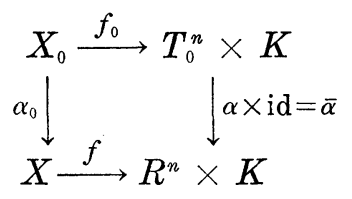

where $X_{0}=\{(x, y) \mid f(x)=\bar{\alpha}(y)\} \subset X \times T_{0}^{n} \times K$ and $\alpha_{0}, f_{0}$ are projection maps. We leave it as an exercise for the reader to prove that $\alpha_{0}$ is also an immersion and that $f_{0}$ is a proper map. Then it is easy to define a PL structure on $X_{0}$ making $X_{0}$ into a polyhedron. (See [17, pp. 76-77] for the definition of a PL structure.) Write $T_{0}^{n}=Y_{0} \cup\left(S^{n-1} \times[0, \infty)\right)$, where $S^{n-1} \times[0, \infty)$ is attached to $Y_{0}$ along $S^{n-1} \equiv S^{n-1} \times\{0\}$ and $e^{n}\left(B_{2}^{n}\right) \subset Y_{0}$. Let $Y_{t}=Y_{0} \cup\left(S^{n-1} \times[0, t]\right)$. With this notation, $T^{n}-\dot{Y}_{t}$ is an $n$-ball.

Assertion. For any $\delta_{1}>0$ we can choose $\delta$ small enough so that $f_{0}$ is a $p^{-1}\left(\delta_{1}\right)$-equivalence over $Y_{3} \times K$.

Proof. Let $g: B_{3}^{n} \times K \rightarrow X$ be a map which is a $p^{-1}(\delta)$-inverse of $f$ over $B_{3}^{n} \times K$. We want to define a map $g_{0}: Y_{3} \times K \rightarrow X_{0}$ which is a $p^{-1}\left(\delta_{1}\right)$-inverse of $f_{0}$ over $Y_{3} \times K$. Choose any $z \in Y_{3} \times K$ and consider $g \circ \bar{\alpha}(z) \in X$. Note that $f \circ g \circ \bar{\alpha}(z)$ is $p^{-1}(\delta)$-close to $\bar{\alpha}(z)$. For any fixed metric on $T_{0}^{n}$ choose $\mu<\delta_{1} / 2$ small enough so that $\alpha \mid B_{\mu}(y)$ is an open embedding, for each $y \in Y_{3}$. Then $\delta$ is chosen small enough so that $B_{\hat{o}}(\alpha(y)) \subset \alpha B_{k}(y)$, for each $y \in Y_{3}$. We therefore define $g_{0}$ by

$$
g_{0}(y, k)=\left(g \circ \bar{\alpha}(y, k),\left(\bar{\alpha} \mid B_{\mu}(y) \times K\right)^{-1}(f \circ g \circ \bar{\alpha}(y, k))\right) .
$$

$g_{0}$ is well-defined because $\operatorname{im}(\bar{\alpha}) \subset B_{3}^{n} \times K=$ domain $(g)$.

To see that $f_{0} \circ g_{0}$ is $p^{-1}\left(\delta_{1}\right)$-homotopic to id we first choose a $p^{-1}(\delta)$-homotopy $\theta_{t}: f \circ g \simeq$ id. Define a homotopy $\bar{\theta}_{t}: Y_{3} \times K \rightarrow T_{0}^{n} \times$ 
$K$ by

$$
\bar{\theta}_{t}(y, k)=\left(\bar{\alpha} \mid B_{\mu}(y) \times K\right)^{-1}\left(\theta_{t} \circ \bar{\alpha}(y, k)\right) .
$$

It is clear that $\bar{\theta}_{t}$ is a $p^{-1}\left(\delta_{1}\right)$-homotopy of $f_{0} \circ g_{0}$ to id.

To see that $g_{0} \circ f_{0} \mid f_{0}^{-1}\left(Y_{3} \times K\right)$ is $\left(p \circ f_{0}\right)^{-1}\left(\delta_{1}\right)$-homotopic to id let $\varphi_{t}: f^{-1}\left(B_{3}^{n} \times K\right) \rightarrow X$ be a $(p \circ f)^{-1}(\delta)$-homotopy of $g \circ f \mid f^{-1}\left(B_{3}^{n} \times K\right)$ to id. Define a homotopy $\bar{\varphi}_{t}: f_{0}^{-1}\left(Y_{3} \times K\right) \rightarrow X_{0}$ by

$$
\bar{\varphi}_{t}(x,(y, k))=\left(\varphi_{t}(x),\left(\bar{\alpha} \mid B_{\mu}(y) \times K\right)^{-1}\left(f \circ \varphi_{1}(x)\right)\right) .
$$

This is a $\left(p \circ f_{0}\right)^{-1}\left(\delta_{1}\right)$-homotopy of $g_{0} \circ f_{0} \mid f_{0}^{-1}\left(Y_{3} \times K\right)$ to id.

Now, since $f_{0}$ is proper we may choose a compact subpolyhedron $X_{1}$ of $X_{0}$ so that

$$
f_{0}^{-1}\left(Y_{3.5} \times K\right) \subset X_{1} \subset f_{0}^{-1}\left(Y_{4} \times K\right) .
$$

Note that if $\delta_{1}$ is small enough, then $f_{1}=f_{0} \mid X_{1}: X_{1} \rightarrow Y_{4} \times K$ is a $p^{-1}\left(\delta_{1}\right)$-equivalence over $Y_{3} \times K$.

B. Capping off to get $T^{n} \times K$. We first construct $f_{3}: X_{3} \rightarrow T^{n} \times$ $K$, a $p^{-1}\left(\delta_{3}\right)$-equivalence which agrees with $f_{1}$ over $Y_{0} \times K$. This is done in two steps.

I. Construction of $X_{2}$. By Theorem 4.3 we can choose $\delta_{1}$ small enough so that we can add a compact polyhedron to $f_{1}^{-1}\left(S^{n-1} \times\right.$ $[2,4] \times K)$ and thereby replace $f_{1}$ by a homotopy equivalence $f_{2}$ : $X_{2} \rightarrow Y_{4} \times K$ which agrees with $f_{1}$ over $Y_{2} \times K$. Moreover we have $f_{2}\left(X_{2}-\dot{X}_{1}\right) \subset\left(Y_{4}-\dot{Y}_{2}\right) \times K$, and $f_{2}$ has a homotopy inverse $g_{2}: Y_{4} \times$ $K \rightarrow X_{2}$ which behaves in the following well-controlled manner (for $\delta_{2}$ small and dependent on $\delta_{1}$ ):

(1) $f_{2} \circ g_{2} \simeq$ id via a homotopy which is a $p^{-1}\left(\delta_{2}\right)$-homotopy on $Y_{2} \times K$, and on $\left(Y_{4}-\dot{\circ}_{2}\right) \times K$ it takes place in $\left(Y_{4}-Y_{1}\right) \times K$.

(2) $g_{2} \circ f_{2} \simeq$ id via a homotopy which is a $\left(p \circ f_{2}\right)^{-1}\left(\delta_{2}\right)$-homotopy on $f_{2}^{-1}\left(Y_{2} \times K\right)$, and on $f_{2}^{-1}\left(\left(Y_{4}-\dot{Y}_{2}\right) \times K\right)$ it takes place in $f_{2}^{-1}\left(\left(Y_{4}-\right.\right.$ $\left.\left.Y_{1}\right) \times K\right)$.

II. Construction of $X_{3}$. Choose a PL map $\beta: S^{n-1} \times K \rightarrow X_{2}$ such that $\beta(x, k)$ is close to $g_{2}(x, 4, k)$, for all $(x, k) \in S^{n-1} \times K$. Form the mapping cylinder $M(\beta)$, which is a polyhedron containing $S^{n-1} \times$ $K$ and $X_{2}$ as subpolyhedra ([10, p. 224]). If $c: M(\beta) \rightarrow X_{2}$ is the collapse to the base, then $f_{2} \circ c: M(\beta) \rightarrow Y_{4} \times K$ is a homotopy equivalence. The restriction $f_{2} \circ c \mid S^{n-1} \times K$ is just $f_{2} \circ \beta: S^{n-1} \times K \rightarrow Y_{4} \times$ $K$. Since $\beta(x, k)$ is close to $g_{2}(x, 4, k)$ we have $f_{2} \circ \beta$ close to $f_{2} \circ g_{2} \mid S^{n-1} \times\{4\} \times K$, which is homotopic to id with the homotopy 
taking place in $\left(Y_{4}-Y_{1}\right) \times K$. By the homotopy extension theorem we have $f_{2} \circ \mathrm{c} \simeq \widetilde{f}_{2}$, where $\widetilde{f}_{2}=f_{2}$ on $X_{2}$ (the base of $\left.M(\beta)\right)$ and $\widetilde{f}_{2}(x, k)=(x, 4, k)$, for all $(x, k) \in S^{n-1} \times K$. Define $X_{3}=M(\beta) \cup\left(T^{n}-\right.$ $\left.\dot{Y}_{4}\right) \times K$, where $\left(T^{n}-\dot{Y}_{4}\right) \times K$ is sewn to $M(\beta)$ by identifying $(x, k)$ in $S^{n-1} \times K \subset M(\beta)$ with $(x, 4, k)$ in $\left(T^{n}-\dot{Y}_{4}\right) \times K$. Then $\widetilde{f}_{2}: M(\beta) \rightarrow$ $Y_{4} \times K$ extends to $f_{3}: X_{3} \rightarrow T^{n} \times K$ by defining $f_{3} \mid X_{3}-M(\beta)=$ id.

Assertion. For every $\delta_{3}^{\prime}>0$ we can choose $\delta_{2}$ small enough so that $f_{3}: X_{3} \rightarrow T^{n} \times K$ has a homotopy inverse $g_{3}: T^{n} \times K \rightarrow X_{3}$ which behaves in the following well-controlled manner:

(1) $f_{3} \circ g_{3} \simeq$ id via a homotopy which is a $p^{-1}\left(\delta_{3}^{\prime}\right)$-homotopy on $Y_{1} \times K$, and on $\left(T^{n}-\dot{Y}_{1}\right) \times K$ it takes place in $\left(T^{n}-\dot{Y}_{0}\right) \times K$.

(2) $g_{3} \circ f_{3} \simeq$ id via a homotopy which is a $\left(p \circ f_{3}\right)^{-1}\left(\delta_{3}^{\prime}\right)$-homotopy on $f_{3}^{-1}\left(Y_{1} \times K\right)$, and on $f_{3}^{-1}\left(\left(T^{n}-\dot{Y}_{1}\right) \times K\right)$ it takes place in $f_{3}^{-1}\left(\left(T^{n}-\right.\right.$ $\left.\left.\dot{\circ}_{0}\right) \times K\right)$.

Proof. Consider the homotopy equivalence $\widetilde{f}_{2}: M(\beta) \rightarrow Y_{4} \times K$ with inverse $g_{2}: Y_{4} \times K \rightarrow X_{2} \hookrightarrow M(\beta)$. Since $\tilde{f}_{2} \mid S^{n-1} \times K: S^{n-1} \times K \rightarrow$ $S^{n-1} \times\{4\} \times K$ is a homeomorphism we can produce a new inverse, $\widetilde{g}_{2}: Y_{4} \times K \rightarrow M(\beta)$, subject to the following restrictions (for $\delta_{3}^{\prime}$ small):

(1) $\widetilde{g}_{2}(x, 4, k)=(x, k)$, for every $(x, 4, k) \in S^{n-1} \times\{4\} \times K$,

(2) $\widetilde{f}_{2} \circ \widetilde{g}_{2} \simeq$ id rel $S^{n-1} \times\{4\} \times K$ via a homotopy which is a $p^{-1}\left(\delta_{3}^{\prime}\right)$-homotopy on $Y_{1} \times K$, and on $\left(Y_{4}-\dot{\circ}_{1}\right) \times K$ it takes place in $\left(Y_{4}-Y_{0}\right) \times K$.

(3) $\widetilde{g}_{2} \circ \widetilde{f}_{2} \simeq$ id rel $S^{n-1} \times K$ via a homotopy which is a $\left(p \circ \widetilde{f}_{2}\right)^{-1}\left(\delta_{3}^{\prime}\right)$-homotopy on $\widetilde{f}_{2}^{-1}\left(Y_{1} \times K\right)$, and on $\widetilde{f}_{2}^{-1}\left(\left(Y_{4}-\dot{Y}_{1}\right) \times K\right)$ it takes place in $\tilde{f}_{2}^{-1}\left(\left(Y_{4}-Y_{0}\right) \times K\right)$.

All of this is a consequence of Proposition 3.2. Then our desired $g_{3}: T^{n} \times K \rightarrow X_{3}$ extends $\widetilde{g}_{2}$ by defining $g_{3}=\mathrm{id}$ on $\left(T^{n}-Y_{4}\right) \times K$.

Using the above Assertion we conclude that if $T^{n}-Y_{0}$ has a small diameter, then $f_{3}$ is a $p^{-1}\left(\delta_{3}\right)$-equivalence. Moreover $\delta_{3}$ can be chosen small corresponding to a small choice of $\delta_{1}$. This completes the construction of $f_{3}$.

To finish step B we must construct $r: T^{n} \times K \times B^{m} \rightarrow X_{3}$. Since $\mathrm{Wh}\left(K \times T^{n}\right)=0$, it follows that the homotopy equivalence $f_{3}$ is a s.h.e. Thus the homotopy inverse $g_{3}: T^{n} \times K \rightarrow X_{3}$ is also a simple homotopy equivalence. It follows from [12] that there is a PL homeomorphism $h$ of $X_{3}$ to a subpolyhedron $X_{3}^{\prime}$ of $T^{n} \times K \times B^{m}$, for some $m \geqq 0$, and a PL collapse $T^{n} \times K \times B^{m} \rightarrow X_{3}^{\prime}$. (See [17, p. 42] for the definition of a PL collapse.) Moreover if $c: T^{n} \times K \times$ $B^{m} \rightarrow X_{3}^{\prime}$ is the contractible PL retraction arising from the collapse, then $f_{3} \circ h^{-1} \circ c: T^{n} \times K \times B^{m} \rightarrow T^{n} \times K$ is homotopic to the projection 
map. Then let $r=h^{-1} \circ c: T^{n} \times K \times B^{m} \rightarrow X_{3}$. It is a contractible PL map for which $f_{3} \circ r$ is homotopic to the projection. By Proposition 2.2 (ii) of $\S 2, f_{3} \circ r: T^{n} \times K \times B^{m} \rightarrow T^{n} \times K$ is a $p^{-1}\left(\delta_{3}\right)$-equivalence. This completes step $B$.

C. Lifting to $R^{n} \times K$. Since $f_{3} \circ r$ is homotopic to the projection, it follows from elementary covering space theory that $f_{3} \circ r$ can be covered by a proper map $f_{4}^{\prime}: R^{n} \times K \times B^{m} \rightarrow R^{n} \times K$ for which there is a bounded homotopy to the projection map. This means that there is a homotopy of $f_{4}^{\prime}$ to proj: $R^{n} \times K \times B^{m} \rightarrow R^{n} \times$ $K$, and $p$ composed with this homotopy yields a bounded homotopy into $R^{n}$. (Recall our convention regarding the map p.) By using an argument similar to that of the Assertion in step A we conclude that $f_{4}^{\prime}$ is a $p^{-1}\left(\varepsilon_{1}\right)$-equivalence (where $\varepsilon_{1}$ is small corresponding to a small choice of $\left.\delta_{3}\right)$. It is easy to check that $\left(e^{n} \times \mathrm{id}\right) \mid:\left(f_{4}^{\prime}\right)^{-1}(U \times$ $K) \rightarrow\left(f_{3} \circ r\right)^{-1}\left(e^{n}(U) \times K\right)$ is $1-1$ and onto, for any open set $U \subset R^{n}$ for which $e^{n} \mid U: U \rightarrow e^{n}(U)$ is $1-1$.

Now choose a large $d$ (to be specified later on) and use the bounded homotopy of $f_{4}^{\prime}$ to the projection map to construct a bounded homotopy of $f_{4}^{\prime}$ to $f_{4}: R^{n} \times K \times B^{m} \rightarrow R^{n} \times K$ for which

(1) $f_{4}=f_{4}^{\prime}$ on $B_{d}^{n} \times K \times B^{m}$,

(2) $q \circ f_{4}=q$ on $\left(R^{n}-B_{d+1}^{n}\right) \times K \times B^{m}$, where $q$ is the projection map to $K$,

(3) $p \circ f_{4}=p \circ f_{4}^{\prime}$.

(The homotopy $f_{4}^{\prime} \simeq f_{4}$ is easily constructed by applying the homotopy $f_{4}^{\prime} \simeq$ proj only in the $K$-coordinate.) If we choose $\left(f_{4}^{\prime}\right)^{-1}\left(B_{2}^{n} \times\right.$ $K) \subset B_{d}^{n} \times K \times B^{m}$, then we see that $f_{4}=f_{4}^{\prime}$ over $B_{2}^{n} \times K$.

Let $\gamma::_{a}^{n} \rightarrow R^{n}$ be a radially-defined homeomorphism which is the identity on $B_{2}^{n}$. Then $f_{5}$ is defined to make the appropriate rectangle commute. The map $f_{6}$ extends $f_{5}$ by defining $f_{6}$ to be the projection map of $\left(R^{n}-\dot{B}_{4}^{n}\right) \times K \times B^{m}$ to $\left(R^{n}-\stackrel{\circ}{B}_{4}^{n}\right) \times K$. This is continuous because $p \circ f_{4}$ is a bounded distance from $p$ and also because $q \circ f_{4}=q$ near $\infty$. We note that $f_{6}=f \circ \alpha_{0} \circ r \circ\left(e^{n} \times\right.$ id $) \circ(\gamma \times \mathrm{id})$ over $B_{2}^{n} \times K$, because $\alpha \circ e^{n} \mid B_{2}^{n}=\mathrm{id}$.

Assertion. For every $\varepsilon>0$ we can choose $\varepsilon_{1}$ small enough so that $f_{6}$ is a $p^{-1}(\varepsilon)$-equivalence.

Proof. We will use Proposition 3.4 by showing that for some $u$ and $v, 0<u<v<4, f_{6}$ is a "small" equivalence over $B_{v}^{n} \times K$ and over $\left(R^{n}-\dot{B}_{u}^{n}\right) \times K$, where the "smallness" is measured in the $R^{n}$-coordinate. Choose $v^{\prime}$ so that $\left(f_{4}^{\prime}\right)^{-1}\left(B_{v^{\prime}}^{n} \times K\right) \subset B_{d}^{n} \times K \times B^{m}$. Then $f_{4}=f_{4}^{\prime}$ over $B_{v^{\prime}}^{n} \times K$ and therefore $f_{4}$ is a $p^{-1}\left(\varepsilon_{1}\right)$-equivalence over $B_{v^{\prime}}^{n} \times K$. If we let $\gamma^{-1}\left(B_{v^{\prime}}^{n}\right)=B_{v^{\prime}}^{n}$ then $f_{6}$ is a $p^{-1}(\varepsilon)$-equivalence 
over $B_{v}^{n} \times K$. We can make $v$ close to 4 by choosing $d$ large and we can make $\varepsilon$ small by choosing $\varepsilon_{1}$ small.

Now for the other half we must prove that $f_{B}$ is a "small" equivalence over $\left(R^{n}-\dot{B}_{u}^{n}\right) \times K$, where $u<v<4$. We will construct a proper map $g_{4}: R^{n} \times K \rightarrow R^{n} \times K \times\{0\} \subset R^{n} \times K \times B^{m}$ so that

(1) $g_{4}$ is a bounded distance from $p$,

(2) $q \circ g_{4}=q$ on the complement of a large compactum,

(3) there are bounded homotopies $\theta_{t}: f_{4} \circ g_{4} \simeq$ id and $\varphi_{t}: g_{4} \circ f_{4} \simeq P$ so that $q \circ \theta_{t}=q$ and $q \circ \varphi_{t}=q$ on the complements of large compacta, where $P(x, k, y)=(x, k, 0)$ and the image of $\varphi_{t}$ lies in $R^{n} \times K \times\{0\}$.

Then define $g_{5}=(\gamma \times \text { id })^{-1} \circ g_{4} \circ(\gamma \times$ id $)$ along with homotopies $\theta_{t}^{\prime}=(\gamma \times \mathrm{id})^{-1} \circ \theta_{t} \circ(\gamma \times \mathrm{id})$ and $\varphi_{t}^{\prime}=(\gamma \times \mathrm{id})^{-1} \circ \varphi_{t} \circ(\gamma \times \mathrm{id})$, which are homotopies of $f_{5} \circ g_{5}$ to id and $g_{5} \circ f_{5}$ to $P$, respectively. By conditions (1) and (2) we see that $g_{5}$ extends via the identity to a map $g_{6}: R^{n} \times$ $K \rightarrow R^{n} \times K \times\{0\}$. Similarly, $\theta_{t}^{\prime}$ extends via the identity to a homotopy $\widetilde{\theta}_{t}: f_{6} \circ g_{6} \simeq \mathrm{id}$. The restriction of $\tilde{\theta}_{t}$ to $\left(R^{n}-\dot{B}_{u}^{n}\right) \times K$ is "small" for $u$ close to 4 . Also $\varphi_{t}^{\prime}$ extends via $P$ to a homotopy of $g_{6} \circ f_{6}$ to $P$, and combining this with a homotopy of $P$ to id we obtain a homotopy $\widetilde{\varphi}_{t}: g_{6} \circ f_{6} \simeq \mathrm{id}$. We have $p \circ \varphi_{t}=p$ on $\left(R^{n}-\stackrel{B}{B}_{4}^{n}\right) \times K \times B^{m}$, so $f_{6} \circ \varphi_{t}$ is a "small" homotopy on $\left(R^{n}-\dot{B}_{u}^{n}\right) \times K \times B^{m}$, for $u$ close to 4. This suffices to prove that $f_{6}$ is a "small" equivalence over $\left(R^{n}-\dot{B}_{u}^{n}\right) \times K$. We now give the details for the construction of $g_{4}$ and the homotopies $\theta_{t}, \varphi_{t}$.

First choose a $p^{-1}\left(\varepsilon_{1}\right)$-inverse $g_{4}^{\prime}: R^{n} \times K \rightarrow R^{n} \times K \times B^{m}$ of $f_{4}^{\prime}$ and collapse out its $B^{m}$-component so that $g_{4}^{\prime}\left(R^{n} \times K\right) \subset R^{n} \times K \times$ $\{0\}$. Let $i_{0}: R^{n} \times K \rightarrow R^{n} \times K \times\{0\}$ be defined by $i_{0}(x, k)=(x, k, 0)$. Then we have bounded homotopies

$$
i_{0} \simeq i_{0} f_{4}^{\prime} g_{4}^{\prime} \simeq i_{0} \pi g_{4}^{\prime}=g_{4}^{\prime} \quad\left(\pi=\text { proj: } R^{n} \times K \times B^{m} \longrightarrow R^{n} \times K\right) .
$$

Using this we can construct a bounded homotopy of $g_{4}^{\prime}$ to a map $g_{4}: R^{n} \times K \rightarrow R^{n} \times K \times B^{m}$ for which $q \circ g_{4}=q$ and $p \circ g_{4}^{\prime}=p \circ g_{4}$. (Recall that $p$ means projection to everything except $K$, and in this case it is to $R^{n} \times B^{m}$.) Moreover, this homotopy affects only the component of $g_{4}^{\prime}$ in the $K$-coordinate.

Since $f_{4} \simeq f_{4}^{\prime}$ and $g_{4} \simeq g_{4}^{\prime}$, we have obvious bounded homotopies $\theta_{t}: f_{4} \circ g_{4} \simeq$ id and $\varphi_{t}: g_{4} \circ f_{4} \simeq P$. However, we want to construct $\theta_{t}$ and $\varphi_{t}$ so that condition (3) above is fulfilled. For this we must do a little more work. Because of the similarity of the cases, we will only give the details for $\theta_{t}$. Let $f_{4}^{\prime \prime}=\left(p \circ f_{4}^{\prime}, q\right): R^{n} \times K \times B^{m} \rightarrow$ $R^{n} \times K$ and let $h_{t}: q \simeq q \circ g_{4}^{\prime}$ be a homotopy. Define a homotopy $\alpha_{t}$ of $R^{n} \times K$ to $R^{n} \times K$ by

$$
\alpha_{t}=\left(p \circ f_{4}^{\prime} \circ\left(p \circ g_{4}^{\prime}, h_{t}\right), q\right) .
$$


Note that $\alpha_{0}=f_{4}^{\prime \prime} \circ g_{4}$ and $\alpha_{1}=\left(p \circ f_{4}^{\prime} \circ g_{4}^{\prime}, q\right)$. If $\theta_{t}^{\prime}: f_{4}^{\prime} \circ g_{4}^{\prime} \simeq$ id is a bounded homotopy, then $\beta_{t}=\left(p \circ \theta_{t}^{\prime}, q\right)$ gives us a bounded homotopy from $\beta_{0}=\alpha_{1}$ to $\beta_{1}=\mathrm{id}$. Thus

$$
f_{4}^{\prime \prime} \circ g_{4}=\alpha_{0} \simeq \alpha_{1}=\beta_{0} \simeq \beta_{1}=\mathrm{id}
$$

is a bounded homotopy of $f_{4}^{\prime \prime} \circ g_{4}$ to id. Finally we only need to notice that there is a bounded homotopy of $f_{4}$ to $f_{4}^{\prime \prime}$ so that $q$ of the homotopy is constantly $q$ on the complement of $B_{d+1}^{n} \times K \times B^{m}$. So this gives a bounded homotopy $f_{4} \circ g_{4} \simeq f_{4}^{\prime \prime} \circ g_{4}$, and using $\left(^{*}\right)$ above we get our desired homotopy $\theta_{t}: f_{4} \circ g_{4} \simeq \mathrm{id}$.

We now enter into the final phase of the proof of the Handle lemma. Our first task is to construct $\tilde{X}$. Let

$$
\begin{aligned}
& \varphi_{1}=\left(e^{n} \times \mathrm{id}\right)(\gamma \times \mathrm{id}) \mid f_{6}^{-1}\left(\stackrel{\circ}{2}_{2}^{n} \times K\right): f_{6}^{-1}\left(\stackrel{\circ}{B}_{2}^{n} \times K\right) \longrightarrow r^{-1} f_{1}^{-1}\left(e^{n}\left(\dot{B}_{2}^{n}\right) \times K\right), \\
& \varphi_{2}=\alpha_{0} \mid f_{1}^{-1}\left(e^{n}\left(\stackrel{\circ}{B}_{2}^{n}\right) \times K\right): f_{1}^{-1}\left(e^{n}\left(\stackrel{\circ}{B}_{2}^{n}\right) \times K\right) \longrightarrow f^{-1}\left(\stackrel{\circ}{B}_{2}^{n} \times K\right),
\end{aligned}
$$

which are easily seen to be PL homeomorphisms. Choose a compact subpolyhedron $C$ of $f_{1}^{-1}\left(e^{n}\left(\dot{B}_{2}^{n}\right) \times K\right)$ which contains $f_{1}^{-1}\left(e^{n}\left(B_{1}^{n}\right) \times K\right)$. Then $\widetilde{X}$ is defined to be the decomposition space $\widetilde{X}=R^{n} \times K \times B^{m} /$ $\mathscr{D}$, where the nondegenerate elements of $\mathscr{D}$ are

$$
\left\{\{x\} \times\{k\} \times B^{m} \mid x \in R^{n}-{\stackrel{\circ}{B^{n}}}^{n} k \in K\right\} \cup\left\{\varphi_{1}^{-1} \circ \boldsymbol{r}^{-1}(x) \mid x \in C\right\} .
$$

Let $\pi: R^{n} \times K \times B^{m} \rightarrow \widetilde{X}$ be the natural quotient map, which is clearly a contractible map as defined in $\S 2$. Now define $\widetilde{f}: \widetilde{X} \rightarrow$ $R^{n} \times K$ by $\widetilde{f}=f_{6} \circ \pi^{-1}$, which is well-defined. By [10, p. 241], $\widetilde{X}$ supports a $\mathrm{PL}$ structure for which $\tilde{f} \mid: \pi\left(\left(R^{n}-B_{5}^{n}\right) \times K \times B^{m}\right) \rightarrow$ $\left(R^{n}-B_{5}^{n}\right) \times K$ is a PL homeomorphism. Also if $\varphi: \widetilde{f}^{-1}\left(\stackrel{\circ}{B}_{1}^{n} \times K\right) \rightarrow$ $f^{-1}\left(\check{B}_{1}^{n} \times K\right)$ is defined by $\varphi=\varphi_{2} \circ r \circ \varphi_{1}$, then $\varphi$ is a PL homeomorphism and $f \circ \varphi=\widetilde{f}$ over $\dot{B}_{1}^{n} \times K$. Finally we leave it as an exercise for the reader to prove that $\tilde{f}$ is a $p^{-1}(\varepsilon)$-equivalence. (If $g_{6}: R^{n} \times K \rightarrow$ $R^{n} \times K \times B^{m}$ is an $\varepsilon$-inverse for $f_{6}$, then $\pi \circ g_{6}: R^{n} \times K \rightarrow \widetilde{X}$ is an $\varepsilon$-inverse for $\widetilde{f}$.)

REMARK. In the sequel it will be convenient to use $\widetilde{f}$ to identify $\tilde{f}^{-1}\left(\left(R^{n}-\dot{B}_{5}^{n}\right) \times K\right)$ with $\left(R^{n}-\dot{B}_{5}^{n}\right) \times K$ and $\varphi$ to identify $\tilde{f}^{-1}\left(\stackrel{\circ}{B}_{1}^{n} \times K\right)$ with $f^{-1}\left(\stackrel{\circ}{1}_{1}^{n} \times K\right)$. In this case, conditions (2) and (3) are replaced by
$(2)^{\prime} \quad \tilde{f}=\mathrm{id}$ over $\left(R^{n}-B_{5}^{n}\right) \times K$,
(3) $)^{\prime} \tilde{f}=f$ over $\dot{B}_{1}^{n} \times K$.

6. The handle theorem. In this section we use the handle 
lemma to establish the handle theorem. In Proposition 6.1 we first establish a weak version of the handle theorem. It is here that we use the inversion idea of [21]. As in $\S 5, K$ will denote a compact polyhedron such that $\mathrm{Wh}\left(K \times T^{n}\right)=0$, all $n \geqq 0$. Also $p: Z \times K \rightarrow Z$ will always denote projection to $Z$.

Proposition 6.1. For each $\varepsilon>0$ there exists $a \delta>0$ so that if $X$ is a polyhedron and $f: X \rightarrow R^{n} \times K$ is a proper map which is a $p^{-1}(\delta)$-equivalence over $B_{3}^{n} \times K$, then there is a polyhedron $X^{\prime}$ and a proper map $f^{\prime}: X^{\prime} \rightarrow R^{n} \times K$ such that

(1) $f^{\prime}$ is a $p^{-1}(\varepsilon)$-equivalence over $B_{2.5}^{n} \times K$,

(2) $f^{\prime}=f$ over $\left(R^{n}-B_{2}^{n}\right) \times K$,

(3) $f^{\prime}=$ id over $\stackrel{\circ}{B}_{1}^{n} \times K$.

(We use the conventions of the Remark following the proof of the handle lemma.)

Proof. For a given $\delta_{1}>0$ we can use the handle lemma to find a $p^{-1}\left(\delta_{1}\right)$-equivalence $f_{1}: X_{1} \rightarrow R^{n} \times K$ such that $f_{1}=$ id over a neighborhood of $\infty$ and $f_{1}=f$ over $\stackrel{B}{B} .5^{n} \times K$. We can extend $f_{1}$ to a map $\widetilde{f}_{1}: \widetilde{X}_{1} \rightarrow S^{n} \times K$ so that $\tilde{f}_{1} \mid \widetilde{X}_{1}-X_{1}$ is a $\mathrm{PL}$ homeomorphism of $\tilde{X}_{1}-X_{1}$ onto $\left(S^{n}-R^{n}\right) \times K$. (We regard $R^{n}$ as $S^{n}-$ \{point\}.) By Proposition 3.4 we conclude that $\widetilde{f}_{1}$ is a $p^{-1}\left(\delta_{1}^{\prime}\right)$-equivalence, for some $\delta_{1}^{\prime}$ which is small corresponding to a small choice of $\delta_{1}$. By restriction we get

$$
\tilde{f}_{1} \mid \widetilde{X}_{1}-f^{-1}(\{0\} \times K): \widetilde{X}_{1}-f^{-1}(\{0\} \times K) \longrightarrow\left(S^{n}-\{0\}\right) \times K,
$$

which is a $p^{-1}\left(\delta_{1}^{\prime}\right)$-equivalence over any chosen compactum in $\left(S^{n}-\right.$ $\{0\}) \times K$ by choosing $\delta_{1}^{\prime}$ correspondingly small. Moreover, $\widetilde{f}_{1} \mid \widetilde{X}_{1}-$ $f^{-1}(\{0\} \times K)$ equals $f$ over $\left(\dot{B}_{2.5}^{n}-\{0\}\right) \times K$.

By the handle lemma, for any $\delta_{2}>0$ we can choose $\delta_{1}^{\prime}$ small enough so that there is a $p^{-1}\left(\delta_{2}\right)$-equivalence $f_{2}: X_{2} \rightarrow\left(S^{n}-\{0\}\right) \times K$ satisfying $f_{2}=$ id over $\left(B^{n}-\{0\}\right) \times K$ and $f_{2}=\widetilde{f}_{1}$ over $\left(S^{n}-B_{2}^{n}\right) \times K$. Consider the restriction

$$
f_{2} \mid: f_{2}^{-1}\left(\left(B_{2.5}^{\circ}-\{0\}\right) \times K\right) \longrightarrow\left(\stackrel{\circ}{B}_{2.5}^{n}-\{0\}\right) \times K,
$$

which is the identity over $\left(B_{1}^{n}-\{0\}\right) \times K$ and which agrees with $f$ over $\left({\stackrel{\circ}{B_{2.5}^{n}}}^{n}-B_{2}^{n}\right) \times K$. The polyhedra $f_{2}^{-1}\left(\left(\stackrel{\circ}{B}_{2.5}^{n}-\{0\}\right) \times K\right), f^{-1}\left(\left(R^{n}-\right.\right.$ $\left.\left.B_{2}^{n}\right) \times K\right)$ and $\dot{B}_{1}^{n} \times K$ can therefore be added together to form a polyhedron $X^{\prime}$. In a natural manner we can define $f^{\prime}: X^{\prime} \rightarrow R^{n} \times K$ which agrees with $f$ over $\left(R^{n}-B_{2}^{n}\right) \times K$, agrees with $f_{2}$ over $\left(\stackrel{B}{2.5}_{2}^{n}-\right.$ $\{0\}) \times K$, and which is the identity over $\dot{B}_{1}^{n} \times K$. By Proposition 
3.4 we conclude that $f^{\prime}$ is a $p^{-1}(\varepsilon)$-equivalence over $B_{2.5}^{n} \times K$.

HANDLE THEOREM. For each $\varepsilon>0$ there exists $a \delta>0$ so that if $f: X \rightarrow R^{n} \times K$ is a proper map which is a $p^{-1}(\delta)$-equivalence over $B_{3}^{n} \times K$, then there exists a proper map $f_{1}: X \times B^{m} \rightarrow R^{n} \times K$, for some $m \geqq 0$, such that

(1) $f_{1}$ is a $p^{-1}(\varepsilon)$-equivalence over $B_{2,5}^{n} \times K$,

(2) $f_{1}=f \circ$ proj over $\left(R^{n}-B_{2}^{n}\right) \times K$,

(3) $f_{1}$ is a contractible PL map over $\stackrel{\circ}{B}_{1}^{n} \times K$.

Proof. Let $f^{\prime}: X^{\prime} \rightarrow R^{n} \times K$ be the map of Proposition 6.1. Choose a compact subpolyhedron $L$ of $X$ so that

$$
f^{-1}\left(B_{2.3}^{n} \times K\right) \subset L \subset f^{-1}\left(\ddot{B}_{2.4}^{n} \times K\right) .
$$

Also $\operatorname{Bd}(L)$ bounds a compact subpolyhedron $L^{\prime}$ of $X^{\prime}$ so that

$$
\left(f^{\prime}\right)^{-1}\left(B_{2.3}^{n} \times K\right) \subset L^{\prime} \subset\left(f^{\prime}\right)^{-1}\left(\dot{B}_{2.4}^{n} \times K\right) .
$$

Assertion 1. There is a homotopy equivalence $\alpha: L \rightarrow L^{\prime}$ such that $\alpha \mid \operatorname{Bd}(L)=\mathrm{id}$.

Proof. Let $g: B_{3}^{n} \times K \rightarrow X$ be a $p^{-1}(\delta)$-inverse of $f$ over $B_{3}^{n} \times K$ and let $g^{\prime}: B_{2.5}^{n} \times K \rightarrow X^{\prime}$ be a $p^{-1}(\varepsilon)$-inverse of $f^{\prime}$ over $B_{2.5}^{n} \times K$. Choose a $\left(p \circ f^{\prime}\right)^{-1}(\varepsilon)$-homotopy $\theta_{t}^{\prime}$ of $g^{\prime} \circ f^{\prime} \mid\left(f^{\prime}\right)^{-1}\left(B_{2.5}^{n} \times K\right)$ to id and define $\alpha: L \rightarrow L^{\prime}$ as follows:

$$
\alpha= \begin{cases}\mathrm{id}, & \text { on } L-f^{-1}\left(\stackrel{\circ}{B}_{2.2}^{n} \times K\right) . \\ \theta_{10 t-21}^{\prime}, & \text { on } f^{-1}\left(\partial B_{t}^{n} \times K\right)=\left(f^{\prime}\right)^{-1}\left(\partial B_{t}^{n} \times K\right), \quad 2.1 \leqq t \leqq 2.2 . \\ g^{\prime} \circ f, & \text { on } f^{-1}\left(B_{2.1}^{n} \times K\right) .\end{cases}
$$

This makes sense provided that $\delta$ and $\varepsilon$ are small. To show that $\alpha$ is a homotopy equivalence we invoke Proposition 3.4. Specifically we show that $\alpha$ is a "small" equivalence over $L^{\prime}-\left(f^{\prime}\right)^{-1}\left(\stackrel{\circ}{2.1}_{2.1}^{n} \times K\right)$ and over $\left(f^{\prime}\right)^{-1}\left(B_{2.2}^{n} \times K\right)$, where the "small" measurement is made in $R^{n}$ upon application of $p \circ f^{\prime}$.

To see that $\alpha$ is an equivalence over $L^{\prime}-\left(f^{\prime}\right)^{-1}\left(\stackrel{\circ}{B}_{2.1}^{n} \times K\right)$ we define $g_{1}: L^{\prime}-\left(f^{\prime}\right)^{-1}\left(B_{2.1}^{n} \times K\right) \rightarrow L$ by $g_{1}=\mathrm{id}$. Using the homotopy $\theta_{t}^{\prime}$ we easily see that $g_{1}$ is an inverse of $\alpha$ over $L^{\prime}-\left(f^{\prime}\right)^{-1}\left(\dot{B}_{2.1}^{n} \times K\right)$. To see that $\alpha$ is an equivalence over $\left(f^{\prime}\right)^{-1}\left(B_{2.2}^{n} \times K\right)$ we define $g_{2}$ : $\left(f^{\prime}\right)^{-1}\left(B_{2.2}^{n} \times K\right) \rightarrow L$ by $g_{2}=g \circ f^{\prime}$. Then

$$
\alpha \circ g_{2}=\alpha \circ g \circ f^{\prime} \simeq g^{\prime} \circ f \circ g \circ f^{\prime} \simeq g^{\prime} \circ f^{\prime} \simeq \mathrm{id},
$$

where the first homotopy arises from $\theta_{t}^{\prime}$, the second from $f \circ g \simeq \mathrm{id}$, 
and the third from $g^{\prime} \circ f^{\prime} \simeq$ id. Similarly,

$$
g_{2} \circ \alpha \mid \alpha^{-1}\left(f^{\prime}\right)^{-1}\left(B_{2.2}^{n} \times K\right) \simeq \mathrm{id} .
$$

It is easy to check the "smallness" condition provided that $\varepsilon$ and $\delta$ are chosen small.

How choose a compact subpolyhedron $L_{1}$ of $L$ so that

$$
f^{-1}\left(B_{2.1}^{n} \times K\right) \subset L_{1} \subset f^{-1}\left(B_{2.2}^{n} \times K\right)
$$

and let $L_{1}^{\prime}$ be the corresponding subpolyhedron of $L^{\prime}$ bounded by $\mathrm{Bd}\left(L_{1}\right)$. It is clear from the proof of Assertion 1 that the homotopy equivalence $\alpha: L \rightarrow L^{\prime}$ may be constructed so that $\alpha \mid L-\stackrel{\circ}{L}_{1}=$ id and $\alpha \mid L_{1}: L_{1} \rightarrow L_{1}^{\prime}$ is also a homotopy equivalence.

Assertion 2. $\alpha$ is a s.h.e.

Proof. The proof is similar to Theorem 4.3. To show that $\alpha$ is a s.h.e. we need to show that its Whitehead torsion $\tau(\alpha)$, which lies in the Whitehead group Wh $\left(L^{\prime}\right)$, vanishes [11, p. 72]. Using the Sum Theorem for Whitehead torsion [11, p. 76] we have

$$
\tau(\alpha)=\tau\left(\alpha \mid L_{1}\right)+\tau\left(\alpha \mid L-\dot{L}_{1}\right)+\tau\left(\alpha \mid \operatorname{Bd}\left(L_{1}\right)\right),
$$

where we have omitted writing down inclusion-induced maps. Now $\alpha \mid L-\check{L}_{1}$ and $\alpha \mid \mathrm{Bd}\left(L_{1}\right)$ are identity maps, so their torsion vanishes. Thus we have $\tau(\alpha)=i_{*} \tau\left(\alpha \mid L_{1}\right)$, where $i$ is the inclusion $L_{1}^{\prime} \hookrightarrow L^{\prime}$ and $i_{*}$ is the induced map on Whitehead groups, $i_{*}: \mathrm{Wh}\left(L_{1}^{\prime}\right) \rightarrow \mathrm{Wh}\left(L^{\prime}\right)$. But $i$ is homotopic to the composition

$$
L_{1}^{\prime} \stackrel{f^{\prime}}{\longrightarrow} B_{2.2}^{n} \times K \stackrel{g^{\prime}}{\longrightarrow}\left(f^{\prime}\right)^{-1}\left(B_{2.3}^{n} \times K\right) \subset L^{\prime},
$$

and since $\mathrm{Wh}(K)=0$ we have $\mathrm{Wh}\left(B_{2.2}^{n} \times K\right)=0$. Thus $i_{*}$ factors through 0 , implying that $i_{*}$ is the 0-map. This gives $\tau(\alpha)=$ $i_{*} \tau\left(\alpha \mid L_{1}\right)=0$.

Using Assertion 2 we can find a compact polyhedron $J$ which collapses to $L$ and a contractible $\mathrm{PL}$ map $u: J \rightarrow L^{\prime}$ such that $u \mid \mathrm{Bd}(L)=\mathrm{id}$. This follows easily from the fact that $\alpha$ is a s.h.e. (See $[11, \mathrm{p} .16]$ for the CW case.) Then $u$ extends to $\tilde{u}: X \cup J \rightarrow X^{\prime}$

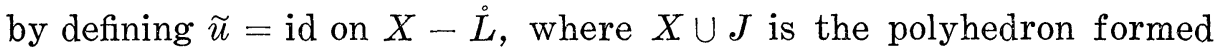
by sewing $X$ to $J$ along $L$. It follows from [12] that any collapse $A \searrow B$ can be reversed to obtain a collapse $B \times B^{m} \searrow A^{\prime}$, for some $m \geqq 0$ and some PL copy $A^{\prime}$ of $A$. Applying this to the collapse $X \cup J \searrow X$ we obtain a contractible PL map $v: X \times B^{m} \rightarrow X \cup J$. It 
follows directly from the proof in [12] that $v$ can be constructed so that it is the projection map from $f^{-1}\left(\left(R^{n}-\stackrel{\circ}{B}_{2.4}^{n}\right) \times B^{m}\right.$ to $f^{-1}\left(\left(R_{n}-\dot{B}_{2.4}^{n}\right) \times K\right)$. Then

$$
f_{1}: X \times B^{m} \stackrel{v}{\longrightarrow} X \cup J \stackrel{\tilde{u}}{\longrightarrow} X^{\prime} \stackrel{f^{\prime}}{\longrightarrow} R^{n} \times K
$$

fulfills our requirements (except that $f_{1}=f \circ$ proj over $\left(R^{n}-B_{2.4}^{n}\right) \times K$ rather than over $\left.\left(R^{n}-B_{2}^{n}\right) \times K\right)$.

7. A splitting theorem. We will use the Handle theorem to establish Theorem 7.2, a result which will be needed in $\S 8$. In Lemma 7.1 we start with a very special case. For notation let $Y$ be a polyhedron with a fixed triangulation and let $\Delta \subset Y$ be a simplex which is not the face of any other simplex. It will be convenient to identify the combinatorial interior of $\Delta$ with $R^{n}$, and we will use $\partial \Delta$ for its combinatorial boundary. Also $K$ and $p: Z \times$ $K \rightarrow Z$ will be as in $\S 6$. Choose an open cover $\gamma$ which contains $R^{n}$ as one of its elements.

Lemma 7.1. For every open cover $\alpha$ of $Y$ there is an open cover $\beta$ of $Y$ so that if $X$ is a polyhedron and $f: X \rightarrow Y \times K$ is a $p^{-1}(\beta)$-equivalence, then there is an $m \geqq 0$, a closed subpolyhedron $X_{1}$ of $X \times B^{m}$, and a proper map $f_{1}: X_{1} \rightarrow\left(Y-R^{n}\right) \times K$ such that

(1) $f_{1}$ is a $p^{-1}\left(\alpha^{\prime}\right)$-equivalence, where $\alpha^{\prime}$ is the restriction of $\alpha$ to $Y-R^{n}$,

(2) $f_{1}$ is $p^{-1}(\alpha)$-homotopic to $f \circ$ proj $\mid X_{1}$ (with the homotopy taking place in $Y \times K)$.

REMARKs. There is also a generalization of this result when $\Delta$ is replaced by a finite union of $n$-simplexes in the given triangulation of $Y$, each of which is not the face of any other simplex. Let $\left\{\Delta_{i}\right\}_{i=1}^{k}$ be this collection of $n$-simplexes, where $\Delta_{i}$ has combinatorial interior $R_{i}^{n}$. Also $\gamma$ is chosen to be any open cover which contains each $R_{i}^{n}$ as one of its elements. The generalization goes as follows: For each $\alpha$ there is a $\beta$ so that each $p^{-1}(\beta)$-equivalence $f: X \rightarrow Y \times$ $K$ yields a proper map $f_{1}: X_{1} \rightarrow\left(Y-\bigcup_{i=1}^{k} R_{i}^{n}\right) \times K\left(\right.$ for $\left.X_{1} \subset X \times B^{m}\right)$ such that

(1) $f_{1}$ is a $p^{-1}\left(\alpha^{\prime}\right)$-equivalence,

(2) $f_{1}$ is $p^{-1}(\alpha)$-homotopic to $f \circ$ proj $\mid X_{1}$.

There are almost no changes in the proof to obtain this generalization. We have treated this special case here only for simplicity of notation.

Proof. By restriction we get a proper map 


$$
f \mid f^{-1}\left(R^{n} \times K\right): f^{-1}\left(R^{n} \times K\right) \longrightarrow R^{n} \times K .
$$

Note that $f \mid f^{-1}\left(R^{n} \times K\right)$ is a $p^{-1}(\varepsilon)$-equivalence over any $B_{r}^{n} \times K$ we choose, for a sufficiently fine choice of $\beta$. Therefore, if $\varepsilon>0$ is given, then the Handle theorem implies that $\beta$ can be chosen fine enough so that there is proper map $f^{\prime}: f^{-1}\left(R^{n} \times K\right) \times B^{m} \rightarrow R^{n} \times$ $K$ which agrees with $f \circ$ proj over $\left(R^{n}-\dot{B}_{r-1}^{n}\right) \times K$, is a $p^{-1}(\varepsilon)$-equivalence over $B_{r}^{n} \times K$, and which is a contractible PL map over $B_{r-2}^{n} \times K$. Then $f^{\prime}$ naturally extends to $\widetilde{f}: X \times B^{m} \rightarrow Y \times K$ by defining $\tilde{f}=f \circ$ proj over $\left(Y-R^{n}\right) \times K$. Our desired $X_{1}$ is defined to be

$$
X_{1}=\left(X \times B^{m}\right)-\tilde{f}^{-1}\left(\stackrel{\circ}{B}_{r-2}^{n} \times K\right) .
$$

Let $s: Y-\{0\} \rightarrow Y-R^{n}$ be a radially-defined retraction and define $f_{1}: X_{1} \rightarrow\left(Y-R^{n}\right) \times K$ to be

$$
f_{1}=(s \times \mathrm{id}) \circ \tilde{f} \mid X_{1}: X_{1} \stackrel{\tilde{f}}{\longrightarrow}(Y-\{0\}) \times K \stackrel{s \times \mathrm{id}}{\longrightarrow}\left(Y-R^{n}\right) \times K .
$$

We must show that $X_{1}$ and $f_{1}$ meet our requirements (1) and (2). We examine them one-by-one.

(1) It is a nontrivial matter to show that $f_{1}$ is a $p^{-1}(\alpha)$-equivalence. (For simplicity, $\alpha^{\prime}$ now becomes $\alpha$.) Let $u$ : $\left(B_{r-2}^{n}-\{0\}\right) \times$ $K \rightarrow \partial B_{r-2}^{n} \times K$ be the radially-defined retraction and let $u_{t}: u \simeq$ id be the radially-defined homotopy of $u$ to id. Since $\widetilde{f} \mid \widetilde{f}^{-1}\left(B_{r-2}^{n} \times K\right)$ is a contractible map we can "lift" $u$ to a retraction $\tilde{u}: \widetilde{f}^{-1}\left(\left(B_{r-2}^{n}-\right.\right.$ $\{0\}) \times K) \rightarrow \widetilde{f}^{-1}\left(\partial B_{r-2}^{n} \times K\right)$ such that $\tilde{f} \circ \tilde{u}$ is as close to $u \circ \widetilde{f}$ as we please. Also $u_{t}$ "lifts" to a homotopy $\tilde{u}_{t}: \tilde{u} \simeq$ id such that $\tilde{f} \circ \tilde{u}_{t}$ is close to $u_{t} \circ \widetilde{f}$ and such that $\tilde{u}_{t} \mid \widetilde{f}^{-1}\left(\partial B_{r-2}^{n} \times K\right)=\mathrm{id}$, for each $t$ (proof same as Proposition 3.1). Then $\widetilde{u}_{t}$ extends trivially to a homotopy $v_{t}: \tilde{f}^{-1}((Y-\{0\}) \times K) \rightarrow X \times B^{m}$ such that $v_{0}$ is a retraction of $\tilde{f}^{-1}((Y-\{0\}) \times K)$ onto $X_{1}$. We are now ready to construct a $p^{-1}(\alpha)$-inverse of $f_{1}$. It follows from Proposition 3.4 that $\widetilde{f}$ is a $p^{-1}\left(\alpha_{1}\right)$-equivalence, where $\alpha_{1}$ is fine corresponding to a fine choice of $\beta$. Let $\tilde{g}: Y \times K \rightarrow X \times B^{m}$ be a $p^{-1}\left(\alpha_{1}\right)$-inverse of $\widetilde{f}$ and define $g_{1}:\left(Y-R^{n}\right) \times K \rightarrow X_{1}$ by $g_{1}=v_{0} \circ \widetilde{g}$. It is now easy to show that $g_{1}$ is a $p^{-1}(\alpha)$-inverse of $f_{1}$. We have

$$
f_{1} \circ g_{1}=(s \times \mathrm{id}) \circ \tilde{f} \circ v_{0} \circ \widetilde{g} \simeq(s \times \mathrm{id}) \circ \tilde{f} \circ \widetilde{g} \simeq s \times \mathrm{id}=\mathrm{id},
$$

where the maps are all restricted to $\left(Y-R^{n}\right) \times K$. The first homotopy comes from $v_{0} \simeq \mathrm{id}$, the second from $\widetilde{f} \circ \widetilde{g} \simeq \mathrm{id}$. We also have

$$
g_{1} \circ f_{1}=v_{0} \circ \widetilde{g} \circ(s \times \mathrm{id}) \circ \widetilde{f} \simeq v_{0} \circ \widetilde{g} \circ \widetilde{f} \simeq v_{0}=\mathrm{id},
$$

where the maps are all restricted to $X_{1}$. The first homotopy comes from the natural radial homotopy $s \simeq \mathrm{id}$, the second from $\widetilde{g} \circ \widetilde{f} \simeq \mathrm{id}$. 
If $r$ is large, then we conclude that $f_{1} \circ g_{1} \simeq$ id is a $p^{-1}(\alpha)$-homotopy and $g_{1} \circ f_{1} \simeq$ id is a $\left(p \circ f_{1}\right)^{-1}(\alpha)$-homotopy. Thus $f_{1}$ is a $p^{-1}(\alpha)$-equivalence. (2) It is clear that

$$
\widetilde{f} \mid: \widetilde{f}^{-1}\left(\left(\Delta-\stackrel{\circ}{B}_{r-2}^{n}\right) \times K\right) \rightarrow \Delta \times K
$$

is homotopic to $f \circ$ proj rel $\tilde{f}^{-1}(\partial \Delta \times K)$. This deformation can be constructed by letting $R:\left(B_{r}^{n}-\{0\}\right) \times K \rightarrow \partial B_{r}^{n} \times K$ be defined via the radial retraction, and using Proposition 2.1 to homotope the identity on $\widetilde{f}^{-1}\left(\left(Y-\stackrel{\circ}{B}_{r-2}^{n}\right) \times K\right)$ to a map which equals $\widetilde{g} R \widetilde{f}$ on $\tilde{f}^{-1}\left(\left(B_{r}^{n}-\dot{B}_{r-2}^{n}\right) \times K\right)$ and is the identity on $\tilde{f}^{-1}\left(\left(Y-R^{n}\right) \times K\right)$. Since $\widetilde{f}=f \circ$ proj over $\left(R^{n}-\stackrel{\circ}{B}_{r-1}^{n}\right) \times K$, we clearly get our desired deformation of $\tilde{f} \mid$ to $f \circ$ proj rel $\widetilde{f}^{-1}(\partial \Delta \times K)$. Since $f_{1}=(s \times$ id $) \circ \tilde{f} \mid X_{1}$ and $s \times \mathrm{id} \simeq \mathrm{id}$, we conclude that $f_{1} \simeq f \circ$ proj in $Y \times K$ as desired.

We are now ready for our main result. For notation let $Y$ be a polyhedron which is written as the union of closed subpolyhedra $Y_{1}$ and $Y_{2}$, where $Y_{1} \cap Y_{2}$ is compact. We also assume $Y_{1}-Y_{2} \neq \varnothing$, $Y_{2}-Y_{1} \neq \varnothing$.

THEOREM 7.2. For each open cover $\alpha$ of $Y$ there exists an open cover $\beta$ of $Y$ so that if $X$ is a polyhedron and $f: X \rightarrow Y \times K$ is a $p^{-1}(\beta)$-equivalence, then there is an $m \geqq 0$, a subdivision of $X \times B^{m}$ into closed subpolyhedra, $X \times B^{m}=X_{1} \cup X_{2}$, and a proper map $f^{\prime}$ : $X \times B^{m} \rightarrow Y \times K$ such that

(1) $f^{\prime} \mid X_{1}: X_{1} \rightarrow Y_{1} \times K$ is a $p^{-1}(\alpha)$-equivalence,

(2) $f^{\prime} \mid X_{2}: X_{2} \rightarrow Y_{2} \times K$ is a $p^{-1}(\alpha)$-equivalence,

(3) $f^{\prime} \mid X_{1} \cap X_{2}: X_{1} \cap X_{2} \rightarrow\left(Y_{1} \cap Y_{2}\right) \times K$ is a $p^{-1}(\alpha)$-equevalence,

(4) $f^{\prime}$ is $p^{-1}(\alpha)$-homotopic to $f \circ$ proj: $X \times B^{m} \rightarrow Y \times K$.

Proof. Let $N \subset Y$ be a compact subpolyhedron containing $Y_{1} \cap$ $Y_{2}$ in its interior. Consider the open set $\stackrel{\circ}{N}-Y_{1}$. If we inductively remove the interiors of simplexes in $N^{\circ}-Y_{1}$ in order of decreasing dimension, by repeatedly applying Lemma 7.1 , we produce a closed subpolyhedron $P_{1}$ of $X \times B^{m}$ and a proper map $f_{1}^{\prime}: P_{1} \rightarrow(Y-(\stackrel{\circ}{N}-$ $\left.\left.Y_{1}\right)\right) \times K$ such that

(1) $f_{1}^{\prime}$ is a $p^{-1}\left(\alpha_{1}\right)$-equivalence (where $\alpha_{1}$ is fine corresponding to $\beta$ fine),

(2) $f_{1}^{\prime}$ is $p^{-1}\left(\alpha_{1}\right)$-homotopic to $f \circ \operatorname{proj} \mid P_{1}$.

Let $X_{1}$ be the subpolyhedron $\left(f_{1}^{\prime}\right)^{-1}\left(Y_{1} \times K\right)$ and note that $f_{1}^{\prime}$ restricts to give a $p^{-1}\left(\alpha_{1}\right)$-equivalence $f_{1}: X_{1} \rightarrow Y_{1} \times K$. Using the same trick on a neighborhood of $\left(Y_{1} \cap N\right) \times K$ in $Y_{1} \times K$ we can produce a compact subpolyhedron $X_{0}$ of $X_{1}$ and a map $f_{0}: X_{0} \rightarrow\left(Y_{1} \cap\right.$ $N) \times K$ which is a $p^{-1}\left(\alpha_{1}\right)$-equivalence and which is $p^{-1}\left(\alpha_{1}\right)$-homotopic 
to $f \circ$ proj $\mid X_{0}$. (For the sake of simplicity we ignore the stabilization of $X_{1}$ by multiplication with some $B^{m_{1}}$, and we assume the homotopies are all controlled by the same cover $\alpha_{1}$ as above.) Then we define $X_{2}$ to be

$$
X_{2}=X_{0} \cup\left(X \times B^{m}-X_{1}\right) .
$$

Since $f_{0}$ is $p^{-1}\left(\alpha_{1}\right)$-homotopic to $f \circ \operatorname{proj} \mid X_{0}$ we can use Proposition 2.1 to find a map $\bar{f}: X \times B^{m} \rightarrow Y \times K$ such that $\bar{f} \mid X_{0}=f_{0}$ and $\bar{f}$ is $p^{-1}\left(\alpha_{1}\right)$-homotopic to $f \circ$ proj. By Proposition $2.2, \bar{f}$ is a $p^{-1}\left(\alpha_{1}^{\prime}\right)$-equivalence, where $\alpha_{1}^{\prime}$ is fine if $\alpha_{1}$ is fine.

If $\alpha_{1}$ is sufficiently fine, then $\bar{f}\left(X_{1}\right) \subset Y_{1} \times K$ and $\bar{f}\left(X_{2}\right) \subset\left(Y_{2} \cup\right.$ $N) \times K$. Moreover, by Proposition 3.4 we conclude that $\bar{f} \mid X_{1}: X_{1} \rightarrow$ $Y_{1} \times K$ and $\bar{f} \mid X_{2}: X_{2} \rightarrow\left(Y_{2} \cup N\right) \times K$ are $p^{-1}\left(\alpha_{1}\right)$-equivalences (where again we use the same cover $\alpha_{1}$ for simplicity). If $N$ is chosen nicely, then $Y_{1} \cap N$ collapses to $Y_{1} \cap Y_{2}$. Let $c: Y_{1} \cap N \rightarrow Y_{1} \cap Y_{2}$ be a contractible retraction arising from this collapse. This can be set up so that $c$ extends to a contractible map of $Y_{1}$ to $Y_{1}$, and $c$ automatically extends to a contractible map of $Y_{2} \cup N$ to $Y_{2}$. Piecing together these extensions we get an extension of $c$ to a contractible map $\tilde{c}: Y \rightarrow Y$, and $f^{\prime}=(\widetilde{c} \times \mathrm{id}) \circ \bar{f}$ is our desired map by use of Proposition 2.2 (ii). Clearly it is proper if $\alpha$ is sufficiently fine.

8. Proof of Theorem 1. We now use Theorem 7.2 to establish Theorem 1. We first treat the compact case.

THEOREM 8.1. For every compact polyhedron $Y$ there is an $\varepsilon>0$ so that for any compact polyhedron $X$ and $p^{-1}(\varepsilon)$-equivalence $f: X \rightarrow Y \times K, f$ must be a s.h.e.

Proof. We induct on the simplexes in a triangulation of $Y$ as follows. If $Y=$ \{point , then $f$ is essentially a homotopy equivalence from $X$ to $K$ and it must therefore be a s.h.e. by the niceness condition on $\pi_{1}$ of each component of $K$. Passing to the inductive step write $Y=Y_{1} \cup \Delta$, where $\Delta$ is an $n$-simplex which is not the face of any other simplex in $Y$ and $Y_{1}$ is the subpolyhedron of $Y$ which meets $\Delta$ in $\partial \Delta$. Assuming the result to be true for $Y_{1}$, we will prove that it is also true for $Y$. This will suffice to prove our result.

By the induction hypothesis, there exists a $\delta>0$ such that any $p^{-1}(\delta)$-equivalence $Z \rightarrow Y_{1} \times K$ is a s.h.e. By Theorem 7.2 we can choose $\varepsilon>0$ so that if $f: X \rightarrow Y \times K$ is a $p^{-1}(\delta)$-equivalence then we can subdivide, $X \times B^{m}=X_{1} \cup X_{2}$, and find a map $f^{\prime}: X \times B^{m} \rightarrow$ $Y \times K$ so that 
(1) $f^{\prime} \mid X_{1}: X_{1} \rightarrow Y_{1} \times K$ is a $p^{-1}(\delta)$-equivalence (where $\delta=\delta(\varepsilon)$ is small),

(2) $f^{\prime} \mid X_{2}: X_{2} \rightarrow \Delta \times K$ is a $p^{-1}(\delta)$-equivalence,

(3) $f^{\prime} \mid X_{1} \cap X_{2}: X_{1} \cap X_{2} \rightarrow \partial \Delta \times K$ is a $p^{-1}(\delta)$-equivalence,

(4) $f^{\prime}$ is $p^{-1}(\delta)$-homotopic to $f \circ$ proj: $X \times B^{m} \rightarrow Y \times K$.

By (4) all we have to do is to prove that $f^{\prime}$ is a s.h.e. (recall that proj: $X \times B^{m} \rightarrow X$ is always s.h.e.). Using the Sum Theorem it suffices to prove that the restrictions in (1), (2), and (3) are all s.h.e.'s, The second and third are s.h.e.'s because of the niceness condition on $\pi_{1}$ of each component of $K$, and because $\Delta, \partial \Delta$ also have nice $\pi_{1}$ 's. The first is a s.h.e. because of our inductive assumption. Thus $f^{\prime}$ is a s.h.e.

Proof of Theorem 1. Given a polyhedron $Y$ we want to prove that there is an open cover $\alpha$ of $Y$ so that any $\alpha$-equivalence $f: X \rightarrow$ $Y \times K$ is a s.h.e. For the first step write $Y=Y_{1} \cup Y_{2} \cup \cdots$, where the $Y_{i}$ are compact subpolyhedra such that $Y_{i} \cap Y_{j}=\varnothing$, for $\mid i-$ $j \mid \geqq 2$. We are going to apply Theorem 7.2 an infinite number of times. Because of this, a fixed $m$ will not suffice for our subdivisions $X \times B^{m}=X_{1} \cup X_{2}$. So we introduce the following notation. Let $R^{\infty}$ denote the direct $\operatorname{limit} \lim \left\{R^{n}\right\}$, where the bonding maps are the injections $R^{n} \stackrel{\times 0}{\rightarrow} R^{n+1}$. Identify $R^{n}$ with the subset $R^{n} \times\{0\}$ of $R^{\infty}$. Then each $X \times B^{m}$ becomes a subpolyhedron of $X \times R^{\infty}$.

Applying Theorem 7.2 there is a subdivision, $X \times B^{m_{2 i}}=X_{2 i} \cup$ $X_{2 i}^{\prime}$, and a proper map $f_{2 i}: X \times B^{m_{2 i}} \rightarrow Y \times K$ so that

(1) $f_{2 i} \mid X_{2 i}: X_{2 i} \rightarrow Y_{2 i} \times K$ is a $p^{-1}\left(\alpha_{2 i}\right)$-equivalence,

(2) $f_{2 i} \mid X_{2 i}^{\prime}: X_{2 i}^{\prime} \rightarrow\left(Y_{1} \cup Y_{2} \cup \cdots \cup Y_{2 i-1} \cup Y_{2 i+1} \cup \cdots\right) \times K$ is a $p^{-1}\left(\alpha_{2 i}\right)$-equivalence,

(3) $f_{2 i} \mid X_{2 i} \cap X_{2 i}^{\prime}: X_{2 i} \cap X_{2 i}^{\prime} \rightarrow\left[\left(Y_{2 i-1} \cup Y_{2 i+1}\right) \cap Y_{2 i}\right] \times K$ is a $p^{-1}\left(\alpha_{2 i}\right)-$ equivalence,

(4) $f_{2 i}$ is $p^{-1}\left(\alpha_{2 i}\right)$-homotopic to $f \circ$ proj: $X \times B^{m_{2 i}} \rightarrow Y \times K$.

Here $\alpha_{2 i}$ is an open cover of $Y$ which can be chosen fine corresponding to a fine choice of $\alpha$.

Now choose compact subpolyhedra $L_{2 i}$ of $X$ so that

(1) $X_{2 i} \subset L_{2 i} \times B^{m_{2 i}}$,

(2) $L_{2 i} \subset f^{-1}\left(Y_{2 i-1} \cup Y_{2 i} \cup Y_{2 i+1}\right)$,

(3) the $L_{2 i}$ are pairwise-disjoint subpolyhedra of $X$.

Form a subpolyhedron $Z$ of $X \times R^{\infty}$ as follows:

$$
Z=X \cup\left(L_{2} \times B^{m_{2}}\right) \cup\left(L_{4} \times B^{m_{4}}\right) \cup \cdots .
$$

We have a natural decomposition $Z=Z_{2} \cup Z_{4} \cup \cdots$, where $Z_{2 i} \cap$ 
$Z_{2 i+2}=X_{2 i}$ and $Z_{2 i} \subset f^{-1}\left(Y_{2 i-3} \cup Y_{2 i-2} \cup \cdots \cup Y_{2 i+1}\right) \times R^{\infty}$. Using Propositions 2.1 and 3.4 we can easily construct a proper map $F: Z \rightarrow$ $Y \times K$ so that

(1) $F \mid X_{2 i}=f_{2 i}: X_{2 i} \rightarrow Y_{2 i} \times K$,

(2) $F \mid Z_{2 i}: Z_{2 i} \rightarrow\left(Y_{2 i-2} \cup Y_{2 i-1} \cup Y_{2 i}\right) \times K$ is a $p^{-1}\left(\varepsilon_{2 i}\right)$-equivalence, where $\varepsilon_{2 i}$ is small,

(3) $F$ is proper homotopic to $f \circ r$, where $r: Z \rightarrow X$ is the contractible $\mathrm{PL}$ retraction defined by $r(x, t)=x$.

Now $f$ factors as follows:

$$
f: X \stackrel{i}{\longrightarrow} Z \stackrel{r}{\longrightarrow} X \stackrel{f}{\longrightarrow} Y \times K .
$$

Since $i$ and $r$ are s.h.e.'s (an easy consequence of the definition), it suffices to prove that $f \circ r: Z \rightarrow Y \times K$ is a s.h.e. By (3) above we only need to prove that $F$ is a s.h.e.

To see that $F$ is a s.h.e. we decompose $Z$ and $Y \times K$ as follows:

$$
Z=Z_{2} \cup Z_{4} \cup Z_{6} \cup \cdots,
$$

$$
Y \times K=\left[\left(Y_{1} \cup Y_{2}\right) \cup\left(Y_{2} \cup Y_{3} \cup Y_{4}\right) \cup\left(Y_{4} \cup Y_{5} \cup Y_{6}\right) \cup \cdots\right] \times K \text {. }
$$

Then $F \mid Z_{2 i}: Z_{2 i} \rightarrow\left(Y_{2 i-2} \cup Y_{2 i-1} \cup Y_{2 i}\right) \times K$ is a s.h.e. by Theorem 8.1 and $F \mid Z_{2 i} \cap Z_{2 i+2}: Z_{2 i} \cap Z_{2 i+2} \rightarrow Y_{2 i} \times K$ is also a s.h.e. by Theorem 8.1. By the Sum Theorem [20, p. 482] we conclude that $F$ is a s.h.e.

9. Proof of Theorem 2. We are given a proper map $f: X \rightarrow$ $Y$ such that $f \times$ id: $X \times Q \rightarrow Y \times Q$ is proper homotopic to a homeomorphism $h: X \times Q \rightarrow Y \times Q$. We want to prove that $f$ is a s.h.e. We first treat the compact case.

Represent $Q$ by the product $\prod_{i=1}^{\infty} I_{i}$, where $I_{i}=[0,1]$, and identify $I^{n}=I_{1} \times \cdots \times I_{n}$ with $I^{n} \times\{(0,0, \cdots)\}$ in $Q$. Consider the map $f^{\prime}: X \rightarrow Y$ defined by

$$
f^{\prime}: X \subset X \times Q \stackrel{h}{\longrightarrow} Y \times Q \stackrel{\text { proj }}{\longrightarrow} Y,
$$

which is certainly homotopic to $f$. So it suffices to prove that $f^{\prime}$ is a s.h.e.

Let $i$ be the inclusion $X \hookrightarrow X \times I^{n}$ and define $u: X \times I^{n} \rightarrow Y$ by

$$
u: X \times I^{n} \rightleftarrows X \times Q \stackrel{h}{\longrightarrow} Y \times Q \stackrel{p}{\longrightarrow} Y,
$$

where $p=$ proj. Then $f^{\prime}=u \circ i$, and since $i$ is clearly a s.h.e. all we have to do is prove that $u$ is a s.h.e. For this we use Theorem 1 by showing if $\alpha$ is the open cover of $Y$ which comes from Theo- 
rem 1 , then $n$ can be chosen large enough so that $u$ is an $\alpha$-equivalence.

Define $v: Y \rightarrow X \times I^{n}$ by

$$
v: Y \subset Y \times Q \stackrel{h^{-1}}{\longrightarrow} X \times Q \stackrel{q}{\longrightarrow} X \times I^{n},
$$

where $q=$ proj. To see that $u \circ v$ is $\alpha$-homotopic to id we first note that $u \circ v=p \circ h \circ q \circ h^{-1} \mid Y$. Using a homotopy $q \simeq$ id which affects only the $Q$-factor we get a homotopy $u \circ v \simeq p \circ h \circ h^{-1} \mid Y=\mathrm{id}$, which must be an $\alpha$-homotopy for $n$ large. To see that $v \circ u$ is $u^{-1}(\alpha)$ homotopic to id we have $v \circ u=q \circ h^{-1} \circ p \circ h \mid X \times I^{n}$. Using a homotopy of $p$ to id which affects only the $Q$-factor we have $v \circ u \simeq$ $q \circ h^{-1} \circ h \mid X \times I^{n}=\mathrm{id}$. This is certainly a $u^{-1}(\alpha)$-homotopy for $n$ large. Thus $u$ is an $\alpha$-equivalence and this completes the proof of the compact case.

For the noncompact case let $w_{i}: X \rightarrow[0,1], i \geqq 1$, be a sequence of PL maps such that for each $x, w_{i}(x)=0$ for $i$ sufficiently large. Define

$$
\tilde{X}=U\left\{\{x\} \times \prod_{i=1}^{\infty}\left[0, w_{i}(x)\right] \mid x \in X\right\} \subset X \times Q .
$$

If this is done properly, then $\tilde{X}$ is a polyhedron which contains $X$ as a subpolyhedron. Moreover, $\tilde{X}$ collapses to $X$, thus $X \hookrightarrow \tilde{X}$ is a s.h.e. For each $t \in I$ let $r_{t}: I \rightarrow[0, t]$ be the retraction which sends $[t, 1]$ to $\{t\}$. Define $q: X \times Q \rightarrow \widetilde{X}$ by

$$
q\left(x,\left(t_{i}\right)\right)=\left(x, r_{w_{i}(x)}\left(t_{i}\right)\right) .
$$

Then $q$ is a retraction which is proper homotopic to id, with a homotopy which affects only the $Q$-factor. This implies that we may repeat the proof of the compact case above by replacing $X \times I^{n}$ with $\tilde{X}$. If the $w_{i}$ are chosen properly, then $u$ is still an $\alpha$-equivalence.

\section{REFERENCES}

1. H. Bass, Algebraic K-theory, W. A. Benjamin, New York, 1968.

2. K. Borsuk, Concerning homotopy properties of compacta, Fund. Math., 62 (1968), 223-254.

3. M. Brown and M. Cohen, A proof that simple-homotopy equivalent polyhedra are stably homeomorphic, Mich. Math. J., 21 (1974), 181-191.

4. T. A. Chapman, Compact Hilbert cube manifolds and the invariance of Whitehead torsion, Bull. Amer. Math. Soc., 79 (1973), 52-56.

5. - Cell-like mappings of Hilbert cube manifolds: Applications to simple homotopy theory, Bull. Amer. Math. Soc., 79 (1973), 1286-1291.

6. - Cell-like mappings, Lecture notes in mathematics, 482 (1973), 230-240.

7. L Lectures on Hilbert cube manifolds, C. B. M. S. Regional Conference

Series in Math. 28, 1976. 
8. T. A. Chapman, Approximating maps into fiber bundles by homeomorphisms, to appear in Rocky Mountain J. Math.

9. T. A. Chapman and Steve Ferry, Obstruction to finiteness in the proper category, preprint.

10. M. Cohen, Simplicial structures and transverse cellularity, Ann. of Math., 85 (1967), 218-245.

11. - A Course in Simple-Homotopy Theory, Springer-Verlag, New York, 1970. 12. P. Dierker, Note on collapsing $K \times I$, where $K$ is a contractible polyhedron, Proc. Amer. Math. Soc., 19 (1968), 425-428.

13. F. T. Farrell and W. C. Hsiang, $A$ formula for $K_{1}\left(R_{\alpha}[T]\right)$, Proc. Symp. Pure Math., 95 (1973), 813-848.

14. Steve Ferry, An immersion of $T^{n}-D^{n}$ into $R^{n}$, l'Enseignement Math., 20 (1974), $12-13$.

15. The homeomorphism group of a compact Hilbert cube manifold is an $A N R$, preprint.

16. William E. Haver, Mappings between ANRs that are fine homotopy equivalences, Pacific J. Math., 58 (1975), 457-461.

17. J. F. P. Hudson, Piecewise Linear Topology, Benjamin, New York, 1969.

18. R. C. Kirby, Lectures on triangulation of manifolds, U.C. L. A. Lecture Notes, Los Angeles, 1969.

19. L. C. Siebenmann, The obstruction to finding a boundary for an open manifold of dimension greater than five, thesis, Princeton University, 1965.

20. - Infinite simple homotopy types, Indag. Math., 32 (1970), 479-495.

21. - Approximating cellular maps by homeomorphisms, Topology, 11 (1972), 271-294.

22. E. H. Spanier, Algebraic Topology, McGraw-Hill, New York, 1966.

23. C. T. C. Wall, Finiteness conditions for CW complexes, Ann. of Math., 81 (1965), $55-69$.

24. J.H.C. Whitehead, Simplicial spaces, nuclei, and m-groups, Proc. London Math. Soc., 45 (1939), 243-327.

Received March 7, 1977 and in revised form June 13, 1978. Supported in part by NSF Grant MCS 76-06929.

UNIVERSITY OF KENTUCKY

LEXINGTON, KY 40506 


\section{PACIFIC JOURNAL OF MATHEMATICS}

\section{EDITORS}

RICHARD ARENS (Managing Editor)

University of California

Los Angeles, California 90024

C. W. CURTIS

University of Oregon

Eugene, OR 97403

C. C. MOORE

University of California

Berkeley, CA 94720
J. DugundjI

Department of Mathematics University of Southern Californı Los Angeles, California 90007

R. Finn and J. Milgram Stanford University Stanford, California 94305

ASSOCIATE EDITORS

E. F. BECKENBACH

B. H. NeUmanN

F. WOLF

K. YoSHIDA

\section{SUPPORTING INSTITUTIONS}

UNIVERSITY OF BRITISH COLUMBIA CALIFORNIA INSTITUTE OF TECHNOLOGY

UNIVERSITY OF CALIFORNIA

MONTANA STATE UNIVERSITY

UNIVERSITY OF NEVADA, RENO

NEW MEXICO STATE UNIVERSITY

OREGON STATE UNIVERSITY

UNIVERSITY OF OREGON
UNIVERSITY OF SOUTHERN CALIFORNIA

STANFORD UNIVERSITY

UNIVERSITY OF HAWAII

UNIVERSITY OF TOKYO

UNIVERSITY OF UTAH

WASHINGTON STATE UNIVERSITY

UNIVERSITY OF WASHINGTON 


\section{Pacific Journal of Mathematics}

\section{Vol. 80, No. $1 \quad$ September, 1979}

Jeroen Bruijning and Jun-iti Nagata, A characterization of covering dimension by

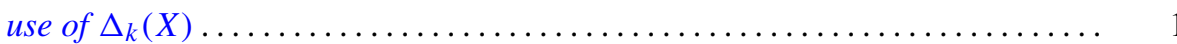

John J. Buoni and Albert Jonathan Klein, On the generalized Calkin algebra ...... 9

Thomas Ashland Chapman, Homotopy conditions which detect simple homotopy

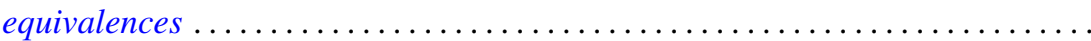

John Albert Chatfield, Solution for an integral equation with continuous interval

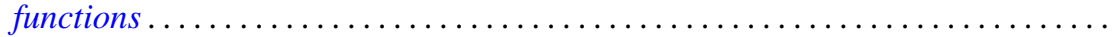

Ajit Kaur Chilana and Ajay Kumar, Spectral synthesis in Segal algebras on

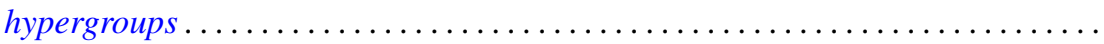

Lung O. Chung, Jiang Luh and Anthony N. Richoux, Derivations and

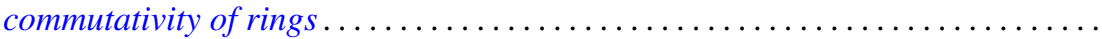

Michael George Cowling and Paul Rodway, Restrictions of certain function spaces to closed subgroups of locally compact groups .....................

David Dixon, The fundamental divisor of normal double points of surfaces........

Hans Georg Feichtinger, Colin C. Graham and Eric Howard Lakien,

Nonfactorization in commutative, weakly selfadjoint Banach algebras . . . . . . .

Michael Freedman, Cancelling 1-handles and some topological imbeddings ....... .

Frank E., III Gerth, The Iwasawa invariant $\mu$ for quadratic fields . . . . . . . . . . . . . .

Maurice Gilmore, Three-dimensional open books constructed from the identity

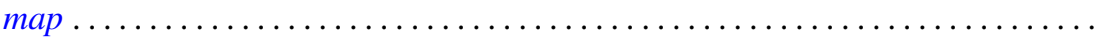

Stanley P. Gudder, A Radon-Nikodým theorem for $*$-algebras .

Peter Wamer Harley, III and George Frank McNulty, When is a point Borel? .

Charles Henry Heiberg, Fourier series with bounded convolution powers . .

Rebecca A. Herb, Characters of averaged discrete series on semisimple real Lie

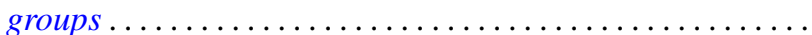

Hideo Imai, On singular indices of rotation free densities . .

Sushil Jajodia, On 2-dimensional CW-complexes with a single 2-cell . . .

Herbert Meyer Kamowitz, Compact operators of the form $u C_{\varphi}$

Matthew Liu and Billy E. Rhoades, Some properties of the Chebyshev method...

213

George Edgar Parker, Semigroups of continuous transformations and generating

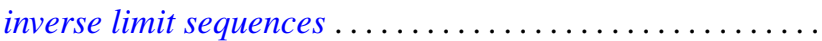

Samuel Murray Rankin, III, Oscillation results for a nonhomogeneous

equation ...

Martin Scharlemann, Transverse Whitehead triangulations ...

Gary Joseph Sherman, A lower bound for the number of conjugacy classes in a

finite nilpotent group

Richard Arthur Shoop, The Lebesgue constants for $\left(f, d_{n}\right)$-summability .

Stuart Jay Sidney, Functions which operate on the real part of a uniform

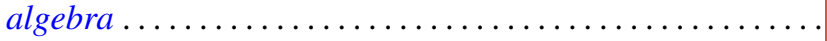

Tim Eden Traynor, The group-valued Lebesgue decomposition 